\title{
Exploration of Properties from both Bulk and Surface of Iron Silicides: A Unified Theoretical Study
}

\author{
Xiaodong Sun, ${ }^{\mathrm{a}, \mathrm{b}}$ Xingwu Liu, ${ }^{\mathrm{c}}{ }^{*}$ Xingchen Liu, ${ }^{\mathrm{a}}$ Yu Meng, ${ }^{\mathrm{e}}$ Chang Song, ${ }^{\mathrm{a}}$ Yong \\ Yang, ${ }^{\text {a,c }}$ Yong-Wang Li, a,c and Xiao-Dong Wen, ${ }^{\mathrm{a}, \mathrm{c}, \mathrm{d}^{*}}$
}

\begin{abstract}
a) State Key Laboratory of Coal Conversion, Institute of Coal Chemistry, Chinese Academy of Sciences, Taiyuan, 030001, China.

b) University of Chinese Academy of Sciences, No.19A Yuquan Road, Beijing, 100049, China.

c) National Energy Center for Coal to Liquids, Synfuels China Co., Ltd, Huairou District, Beijing, 101400, China.

d) Beijing Advanced Innovation Center for Materials Genome Engineering, IndustryUniversity Cooperation Base between Beijing Information S\&T University and Synfuels China Co. Ltd, Beijing, 100101, China.

e) Shaanxi Key Laboratory of Low Metamorphic Coal Clean Utilization, School of Chemistry and Chemical Engineering, Yulin University, Yulin, 719000, China.
\end{abstract}

\section{Table of Contents}

- The comparison of calculation methods.

- Figure S1. The calculation results by different methods.

- Table S1. The key parameters and calculated results for the cell optimization.

- Table S2. Magnetic moment $\left(\mu_{B}\right)$ of every Fe-Si phase.

- The slab models.

- Table S3. The investigated surfaces for the Fe-Si phases. 
- Ab initio atomistic thermodynamics.

- Table S4. The boundary of $\mu_{\mathrm{Si}}$ for Fe-Si phases.

- Figure S2. The average number of neighbors at different distances in the unit cell.

- Table S5. Thermodynamic parameters for $\mathrm{SiH}_{4}$ and $\mathrm{H}_{2}$.

- Table S6. The value of $\mu_{\mathrm{Si}}$ under given conditions.

- Table S7. Surface energies of $\mathrm{Fe}_{3} \mathrm{Si}$.

- Table S8. Surface energies of $\mathrm{Fe}_{2} \mathrm{Si}$.

- Table S9. Surface energies of $\mathrm{Fe}_{5} \mathrm{Si}$.

- Table S10. Surface energies of FeSi.

- Table S11. Surface energies of $\alpha-F e S i 2$.

- Table S12. Surface energies of $\beta-\mathrm{FeSi}_{2}$

- Figure S3. The relationship of surface energy of the Fe ${ }_{3} \mathrm{Si}$ with $\mu \mathrm{Si}$.

- Figure S4. The relationship of surface energy of the $\mathrm{Fe}_{2} \mathrm{Si}$ with $\mu_{\mathrm{Si}}$.

- Figure S5. The relationship of surface energy of the $\mathrm{Fe}_{5} \mathrm{Si} 3$ with $\mu \mathrm{Si}$.

- Figure S6. The relationship of surface energy of the FeSi with $\mu_{\text {Si. }}$

- Figure S7. The relationship of surface energy of the $\alpha-\mathrm{FeSi}_{2}$ with $\mu$ Si.

- Figure S8. The relationship of surface energy of the $\beta-\mathrm{FeSi}_{2}$ with $\mu \mathrm{Si}$.

- Figure S9. Surface structures of most stable surfaces of the iron silicides.

- Figure S10. The average magnetic moment with different cutoff distance.

1. The comparison of calculation methods

All calculations were performed at the density functional level of theory with the Vienna ab initio simulation package (VASP) ${ }^{1-2}$. In order to choose a suitable calculation method we tested the exchange-correlation functional which is treated by the Local Density Approximation (LDA) ${ }^{3}$ and the generalized gradient approximation (GGA) of Perdew and Wang ${ }^{4}$. Within the generalized gradient approximation, the electron-ion interaction was described by the Perdew-Burke-Ernzerhof scheme (PBE) and PW915. 
Here, we choose the formation enthalpy $-\Delta \mathrm{H}_{\mathrm{f}}$ of every Fe-Si phases and the magnetic moment $\left(\mu_{\mathrm{B}}\right)$ as the standards. The tested methods contain PAW-LDA, GGA-PBE, GGA-PW91. And the calculation results are listed in Figure S1. In the Figure S1, the experiments results originate from the reference article $^{6}$ are also listed. The Table S2 lists the calculation and experiment results of the $\mu_{\mathrm{B}}$, the calculation results originate from the reference article ${ }^{7}$.

As the Figure S1 shows, all the calculation results are larger than experiments results for every Fe-Si phase, but the PAW-LDA calculation results are larger than that of the others method. The GGA-PBE and GGA-PW91 methods predict almost the same result for $-\Delta \mathrm{H}_{\mathrm{f}}$, although these two methods calculation results are also larger than experiment results, but they are the relatively accurate methods.

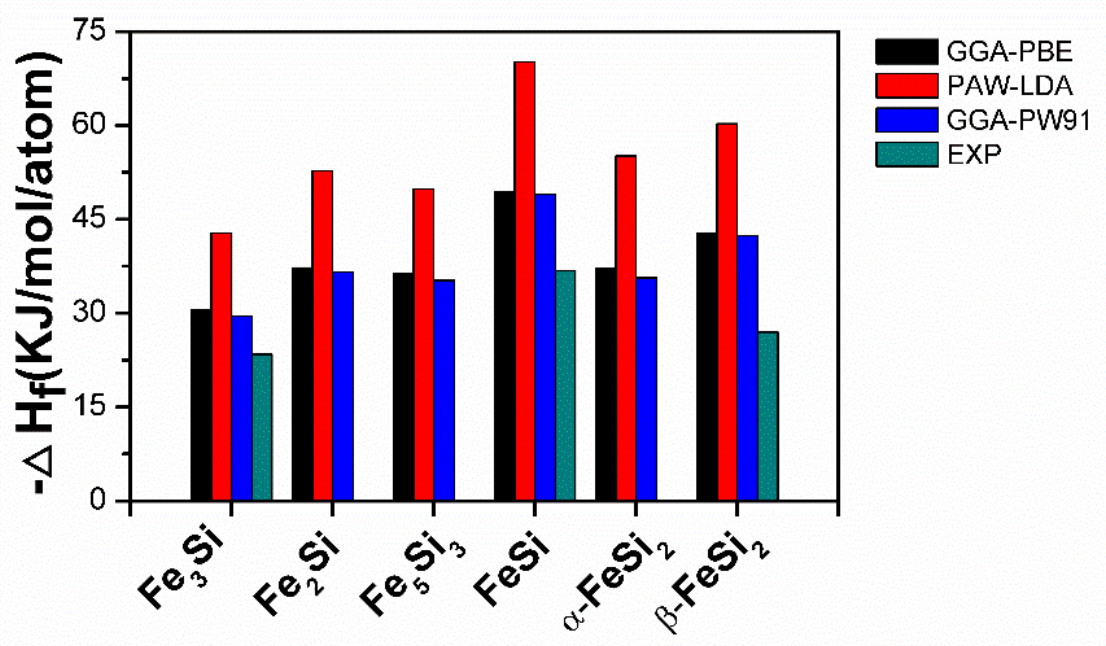

Figure S1. The calculation results of PAW-LDA, GGA-PBE, GGA-PW91. Black rod, red rod, blue rod and green rod represent the results of GGA-PBE, PAW-LDA, GGA-PW91 and the experiment ${ }^{6}$.

As the Table $\mathbf{S} 2$ shows, the local magnetic moment and magnetic moment of $\alpha-$ $\mathrm{Fe}$ and $\mathrm{Fe}_{3} \mathrm{Si}$ which are predicted by the GGA-PBE and GGA-PW91 methods are consistent with the experiment ${ }^{6}$ and calculation results ${ }^{7}$, the deviation is small, in addition, the magnetic moments of the other phases predicted by these two methods are consistent with the calculation results. But, the PAW-LDA method calculation results of the local magnetic moments and magnetic moments are far from the experiment and the calculation results of the reference article ${ }^{7}$. 
The above analysis shows that GGA-PW91 and GGA-PBE are two kinds of effective methods to calculate the $\mathrm{Fe}-\mathrm{Si}$ system, their calculation results are more accurate than that of PAW-LDA. In the following calculation, we choose GGA-PBE as the calculation method.

Table S1. Monkhorst-Pack grid of k-points used for the cell optimization, the calculation result and experiment value of lattice parameters, the temperature ranges for the stable iron silicide ${ }^{6}$.

\begin{tabular}{|c|c|c|c|c|c|}
\hline \multirow{2}{*}{$\begin{array}{l}\text { System } \\
\text { bcc-Fe }\end{array}$} & \multicolumn{3}{|c|}{ Lattice paramaters $(\AA)$} & \multirow{2}{*}{$\frac{\text { k-points }}{14 \times 14 \times 14}$} & \multirow{2}{*}{$\frac{\mathrm{T}(\mathrm{K})}{-}$} \\
\hline & A & $2.867^{\mathrm{a}}$ & $2.823^{\mathrm{b}}$ & & \\
\hline $\mathrm{Fe}_{3} \mathrm{Si}$ & A & $5.644^{\mathrm{a}}$ & $5.591^{b}$ & $7 \times 7 \times 7$ & - \\
\hline \multirow[t]{2}{*}{$\mathrm{Fe}_{2} \mathrm{Si}$} & A & $4.052^{\mathrm{a}}$ & $3.929^{\mathrm{b}}$ & $11 \times 11 \times 8$ & $1363 \sim 1473^{b}$ \\
\hline & $\mathrm{c}$ & $5.086^{\mathrm{a}}$ & $4.930^{\mathrm{b}}$ & & \\
\hline \multirow[t]{2}{*}{$\mathrm{Fe}_{5} \mathrm{Si}_{3}$} & $\mathrm{a}$ & $6.742^{\mathrm{a}}$ & $6.692^{b}$ & $7 \times 7 \times 9$ & $>1098^{\mathrm{b}}$ \\
\hline & $\mathrm{c}$ & $4.708^{\mathrm{a}}$ & $4.667^{b}$ & & \\
\hline $\mathrm{FeSi}$ & $\mathrm{a}$ & $4.483^{\mathrm{a}}$ & $4.439^{b}$ & $9 \times 9 \times 9$ & - \\
\hline \multirow[t]{3}{*}{$\beta-\mathrm{FeSi}_{2}$} & $\mathrm{a}$ & $9.863^{\mathrm{a}}$ & $9.857^{\mathrm{b}}$ & $4 \times 5 \times 5$ & \\
\hline & $\mathrm{b}$ & $7.791^{\mathrm{a}}$ & $7.765^{\mathrm{b}}$ & & \\
\hline & $\mathrm{c}$ & $7.833^{\mathrm{a}}$ & $7.804^{\mathrm{b}}$ & & \\
\hline \multirow[t]{2}{*}{$\alpha-\mathrm{FeSi}_{2}$} & $\mathrm{a}$ & $2.684^{\mathrm{a}}$ & $2.701^{b}$ & $15 \times 15 \times 8$ & $>1210^{\mathrm{b}}$ \\
\hline & $\mathrm{c}$ & $5.128^{\mathrm{a}}$ & $5.132^{b}$ & & \\
\hline $\mathrm{Si}$ & $\mathrm{a}$ & $5.431^{\mathrm{a}}$ & $5.413^{\mathrm{b}}$ & $7 \times 7 \times 7$ & - \\
\hline
\end{tabular}

${ }^{a}$ reference ${ }^{6}$

${ }^{\mathrm{b}}$ this work result 


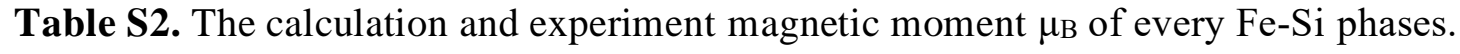
EXP represents the experiment results of reference article ${ }^{6}$, Ref represents the calculation results of reference article ${ }^{7}$.

\begin{tabular}{|c|c|c|c|c|c|c|c|c|c|}
\hline \multirow{3}{*}{ Compound } & & \multicolumn{4}{|c|}{ Local magnetic moment $\mathrm{M}\left(\mu_{\mathrm{B}}\right)$} & \multicolumn{4}{|c|}{ Magnetic moment $\mathrm{M}(\mu \mathrm{B} / \mathrm{Fe}$-atom $)$} \\
\hline & & GGA- & GGA- & PAW- & & GGA- & GGA- & PAW- & \\
\hline & & PBE & PW91 & LDA & $\mathrm{EXP}^{6}$ & PBE & PW91 & LDA & $\operatorname{Ref}^{7}$ \\
\hline \multirow[t]{2}{*}{$\alpha-\mathrm{Fe}$} & & 2.232 & 2.168 & 1.904 & 2.220 & 2.232 & 2.168 & 1.904 & 2.200 \\
\hline & $\mathrm{Fe} 1$ & 2.554 & 2.560 & 2.279 & $2.2-2.4$ & & & & \\
\hline \multirow[t]{2}{*}{$\mathrm{Fe}_{3} \mathrm{Si}$} & $\mathrm{Fe} 2$ & 1.330 & 1.310 & 0.802 & 1.350 & 1.738 & 1.685 & 1.294 & 1.690 \\
\hline & $\mathrm{Si}$ & -0.040 & -0.037 & -0.009 & -0.070 & & & & \\
\hline $\mathrm{Fe}_{2} \mathrm{Si}$ & - & - & - & - & - & 1.052 & & 0.945 & 1.000 \\
\hline $\mathrm{Fe}_{5} \mathrm{Si}_{3}$ & - & - & - & - & - & 1.627 & 1.628 & 1.165 & 1.500 \\
\hline $\mathrm{FeSi}$ & - & - & - & - & - & 0.000 & 0.000 & 0.000 & 0.000 \\
\hline$\beta-\mathrm{FeSi}_{2}$ & - & - & - & - & - & 0.000 & 0.000 & 0.000 & 0.000 \\
\hline$\alpha-\mathrm{FeSi}_{2}$ & - & - & - & - & - & 0.000 & 0.000 & 0.000 & 0.000 \\
\hline $\mathrm{Si}$ & - & - & - & - & - & 0.000 & 0.000 & 0.000 & 0.000 \\
\hline
\end{tabular}




\section{The slab models}

Table S3. The investigated surfaces for the $\mathrm{Fe}_{3} \mathrm{Si}_{1}, \mathrm{Fe}_{2} \mathrm{Si}_{1}, \mathrm{Fe}_{5} \mathrm{Si}_{3}, \mathrm{FeSi}, \alpha-\mathrm{FeSi}_{2}, \beta-\mathrm{FeSi}_{2}$ phases and the corresponding value of k-points.

\begin{tabular}{|c|c|c|c|c|c|c|c|}
\hline \multirow{2}{*}{$\mathrm{Fe}_{3} \mathrm{Si}$} & surface & $(100)$ & $(110)$ & $(111)$ & $(211)$ & $(311)$ & (310) \\
\hline & Kpoints & $3 * 3 * 1$ & $3 * 7 * 1$ & $7 * 7 * 1$ & $3 * 3 * 1$ & $4 * 7 * 1$ & $4 * 3 * 1$ \\
\hline \multirow{4}{*}{$\mathrm{Fe}_{2} \mathrm{Si}$} & surface & $(001)$ & $(110)$ & $(011)$ & $(100)$ & $(101)$ & (111) \\
\hline & Kpoints & $7 * 7 * 1$ & $5 * 4 * 1$ & $7 * 5 * 1$ & $6 * 5 * 1$ & $5 * 7 * 1$ & $5 * 5 * 1$ \\
\hline & surface & $(102)$ & $(104)$ & $(21-2)$ & - & - & - \\
\hline & Kpoints & $3 * 6 * 1$ & $3 * 7 * 1$ & $3 * 5 * 1$ & - & - & - \\
\hline \multirow{4}{*}{$\mathrm{Fe}_{5} \mathrm{Si}_{3}$} & surface & $(001)$ & $(01-2)$ & $(1-2-2)$ & $(2-1-1)$ & $(3-1-3)$ & $(3-11)$ \\
\hline & Kpoints & $5 * 5 * 1$ & $3 * 5 * 1$ & $3 * 3 * 1$ & $3 * 3 * 1$ & $3 * 3 * 1$ & $3 * 3 * 1$ \\
\hline & surface & $(011)$ & $(100)$ & $(110)$ & $(120)$ & $(231)$ & - \\
\hline & Kpoints & $5 * 3 * 1$ & $5 * 5 * 1$ & $5 * 3 * 1$ & $5 * 3 * 1$ & $3 * 3 * 1$ & - \\
\hline \multirow{4}{*}{$\mathrm{FeSi}$} & surface & $(100)$ & $(110)$ & (111) & $(120)$ & $(210)$ & (211) \\
\hline & Kpoints & $7 * 7 * 1$ & $7 * 5 * 1$ & $5 * 5 * 1$ & $7 * 3 * 1$ & $7 * 3 * 1$ & $3 * 5 * 1$ \\
\hline & surface & (311) & $(123)$ & & - & - & - \\
\hline & Kpoints & $3 * 5 * 1$ & $3 * 3 * 1$ & & - & - & - \\
\hline \multirow{4}{*}{$\alpha-\mathrm{FeSi}_{2}$} & surface & $(001)$ & $(101)$ & $(110)$ & $(10-2)$ & $(111)$ & (100) \\
\hline & Kpoints & $9 * 9 * 1$ & $5 * 9 * 1$ & $5 * 7 * 1$ & $9 * 3 * 1$ & $7 * 5 * 1$ & $9 * 5 * 1$ \\
\hline & surface & (113) & $(122)$ & $(114)$ & & - & - \\
\hline & Kpoints & $7 * 3 * 1$ & $5 * 5 * 1$ & $7 * 3 * 1$ & & - & - \\
\hline \multirow{6}{*}{$\beta-\mathrm{FeSi}_{2}$} & surface & $(001)$ & $(010)$ & $(011)$ & $(041)$ & $(114)$ & (211) \\
\hline & Kpoints & $5 * 5 * 1$ & $3 * 3 * 1$ & $3 * 3 * 1$ & $3 * 3 * 1$ & $5 * 3 * 1$ & $3 * 3 * 1$ \\
\hline & surface & $(100)$ & $(101)$ & $(110)$ & $(012)$ & (131) & (331) \\
\hline & Kpoints & $3 * 3 * 1$ & $3 * 3 * 1$ & $3 * 5 * 1$ & $3 * 3 * 1$ & $3 * 3 * 1$ & $5 * 3 * 1$ \\
\hline & surface & (312) & $(421)$ & $(771)$ & & & \\
\hline & Kpoints & $3 * 3 * 1$ & $3 * 3 * 1$ & $3 * 3 * 1$ & & & \\
\hline
\end{tabular}

\section{Ab initio atomistic thermodynamics}

To evaluate the surface stability at different temperature, partial $\mathrm{SiH}_{4}$ pressure, and gas atmosphere, the $a b$ initio atomistic thermodynamics developed by Reuter and Scheffler ${ }^{8-9}$ was used. In this method, the surface free energy $(\gamma)$ of $\mathrm{Fe}_{\mathrm{x}} \mathrm{Si}_{\mathrm{y}}$ can be calculated from Eq. (1), where $\mathrm{G}_{\text {slab }}$ is the Gibbs free energy of a slab with two equivalent surfaces, $\mu \mathrm{Fe}$ and $\mu \mathrm{Si}$ are the chemical potentials of $\mathrm{Fe}$ and $\mathrm{Si}$ atom, respectively, $\mathrm{N}_{\mathrm{Fe}}$ and $\mathrm{N}_{\mathrm{Si}}$ are the numbers of $\mathrm{Fe}$ and $\mathrm{Si}$ atoms in slabs, and $\mathrm{A}$ is the area of surface unit cell.

$\gamma(\mathrm{T}, \mathrm{P})=\frac{1}{2 A}\left(G_{s l a b}\left(\mathrm{~T}, \mathrm{p}, N_{F e}, N_{S i}\right)-N_{F e} \mu_{F e}(\mathrm{~T}, \mathrm{p})-N_{S i} \mu_{S i}(\mathrm{~T}, \mathrm{p})\right)$ 
The chemical potentials of $\mathrm{x} F e$ atoms and $\mathrm{y} \mathrm{C}$ atoms are related to the Gibbs free energy $\left(g_{\text {FexSiy }}^{\text {bulk }}\right)$ per formula unit of the $\mathrm{Fe}_{\mathrm{x}} \mathrm{Si}_{\mathrm{y}}$ bulk as shown in the following equation:

$g_{\text {FexSiy }}^{\text {bulk }}(T, P)=x \mu_{F e}(T, P)+y \mu_{S i}(T, P)$

Inserting this constraint into Eq. (1) leads to Eq. (3) in which the surface free energy depends only on $\mu \mathrm{Si}$, which relates to gas atmosphere, temperature, and pressure (see detailed discussion the relationship of $\mathrm{T}, \mathrm{P}_{\mathrm{SiH} 4}$ to the gas $\left.\mu_{\mathrm{Si}}\right)$.

$\gamma(\mathrm{T}, \mathrm{P})=\frac{1}{2 A}\left(G_{\text {slab }}\left(\mathrm{T}, \mathrm{p}, N_{F e}, N_{S i}\right)-\frac{N_{F e}}{x} g_{F \operatorname{bexSiy}}^{\text {bulk }}(\mathrm{T}, \mathrm{p})-\left(N_{S i}-\right.\right.$ $\left.\left.\frac{y}{x} N_{F e}\right) \mu_{S i}(\mathrm{~T}, \mathrm{p})\right)$

Noteworthy, $\mu$ Si cannot be varied without boundary ${ }^{8-9}$. If $\mu_{\mathrm{Si}}$ became too low, all Fe would leave the sample, that is, the $\mathrm{Fe}_{\mathrm{x}} \mathrm{Si}_{\mathrm{y}}$ phase would decompose into metallic iron, and, if the $\mu \mathrm{Si}$ becomes too high the silicon would leave the sample, that is the $\mathrm{Fe}_{\mathrm{x}} \mathrm{Si}_{\mathrm{y}}$ phase would decompose into silicon crystal, thus the minimum and maximum $\mu_{\mathrm{Si}}$ can be obtained from:

$g_{\text {FexSiy }}^{\text {bulk }}(0,0)-x g_{F e}^{\text {bulk }}(0,0) \leq y \mu_{S i}(0,0)$

$\mu_{S i}(0,0) \leq g_{S i}^{\text {bulk }}(0,0)$

Table S4 lists the boundary of every $\mathrm{Fe}_{x} \mathrm{Si}_{\mathrm{y}}$ phases, during which the $\mathrm{Fe}_{\mathrm{x}} \mathrm{Si}_{\mathrm{y}}$ can be stably exist.

In this paper, all the study will be discussed during this boundary. And this is considered from the thermodynamics; the kinetic parameter, which may play an important role in the silicon deposition on the surface, is not taken into account. 
Table S4. The boundary of $\mu \mathrm{Si}$ for every $\mathrm{Fe}_{x} \mathrm{Si}_{\mathrm{y}}$ phase stably exist.

\begin{tabular}{ccc|ccc}
\hline & $\mathrm{Min} \mu \mathrm{Si}$ & $\mathrm{Max} \mu \mathrm{Si}$ & & $\mathrm{Min} \mu_{\mathrm{Si}}$ & $\mathrm{Max} \mu_{\mathrm{Si}}$ \\
\hline $\mathrm{Fe}_{3} \mathrm{Si}$ & -6.69 & -5.425 & $\mathrm{FeSi}$ & -6.447 & -5.425 \\
$\mathrm{Fe}_{2} \mathrm{Si}$ & -6.584 & -5.425 & $\beta-\mathrm{FeSi}_{2}$ & -6.091 & -5.425 \\
$\mathrm{Fe}_{5} \mathrm{Si}_{3}$ & -6.424 & -5.425 & $\alpha-\mathrm{FeSi}_{2}$ & -6.091 & -5.425 \\
\hline
\end{tabular}

\section{The relationship of $\mathrm{T}, \mathrm{P}_{\mathrm{SiH} 4}$ to the gas $\mu_{\mathrm{Si}}$}

For iron silicification under $\mathrm{SiH}_{4} / \mathrm{H}_{2}$, the way to generate overall surface iron silicides is $\mathrm{SiH}_{4}$ disproportionation into $\mathrm{Si}$ and $\mathrm{H}_{2}\left(\mathrm{SiH}_{4}=\mathrm{Si}+2 \mathrm{H}_{2}\right)^{10}$. The $\mu \mathrm{Si}$ is determined by the equilibrium of $\mathrm{SiH}_{4}$ and $\mathrm{H}_{2}$ gas mixture as shown in the following equation:

$\mathrm{SiH}_{4}=\mathrm{Si}+2 \mathrm{H}_{2}$

$\mu_{S i}=\mu_{S i H 4}-2 \mu_{H 2}$

For each ideal gas, its $\mu_{\mathrm{i}}$ correlates with temperature and pressure is presented in Eq. (7); $\mathrm{E}_{\mathrm{i}}(0 \mathrm{~K})$ is the total energy of the ideal gas molecule; $\tilde{\mu}_{i}\left(T, p^{\theta}\right)$ includes the thermal contributions of the ideal gas molecule and the ideal gas molecule entropy at 1 atm, as shown in Eq.(8), which has been taken from the standard thermodynamic tables ${ }^{11}$ and are given in the Table S5.

$\mu_{i}\left(\mathrm{~T}, \mathrm{P}_{i}\right)=E_{i}(0 \mathrm{k})+\widetilde{\mu_{\imath}}\left(\mathrm{T}, \mathrm{p}^{\theta}\right)+\mathrm{k}_{B} T \ln \left(\frac{\mathrm{p}_{\mathrm{i}}}{\mathrm{p}^{\theta}}\right)$

$\widetilde{\mu_{\iota}}\left(\mathrm{T}, \mathrm{p}^{\theta}\right)=\left[\mathrm{H}\left(\mathrm{T}, p^{\theta}\right)-H^{\theta}\left(T_{r}\right)\right]-\mathrm{T}\left[S^{\theta}(\mathrm{T})-S^{\theta}(0 \mathrm{~K})\right], p^{\theta}=0.1 M p a, T_{r}=$ $298 K$

Inserting Eq. (7) into Eq. (6), the $\mu_{\mathrm{Si}}$ under $\mathrm{SiH}_{4} / \mathrm{H}_{2}$ pretreatment can be expressed in Eq. (9).

$\mu_{S i}=\left[E_{S i H 4}(0 K)-2 E_{H 2}(0 K)\right]+\left[\tilde{\mu}_{S i H 4}\left(T, p^{\theta}\right)-2 \tilde{\mu}_{H 2}\left(T, p^{\theta}\right)\right]+k_{B} T \ln \left(\frac{p_{S i H 4} p^{\theta}}{p_{H 2}^{2}}\right)$ 

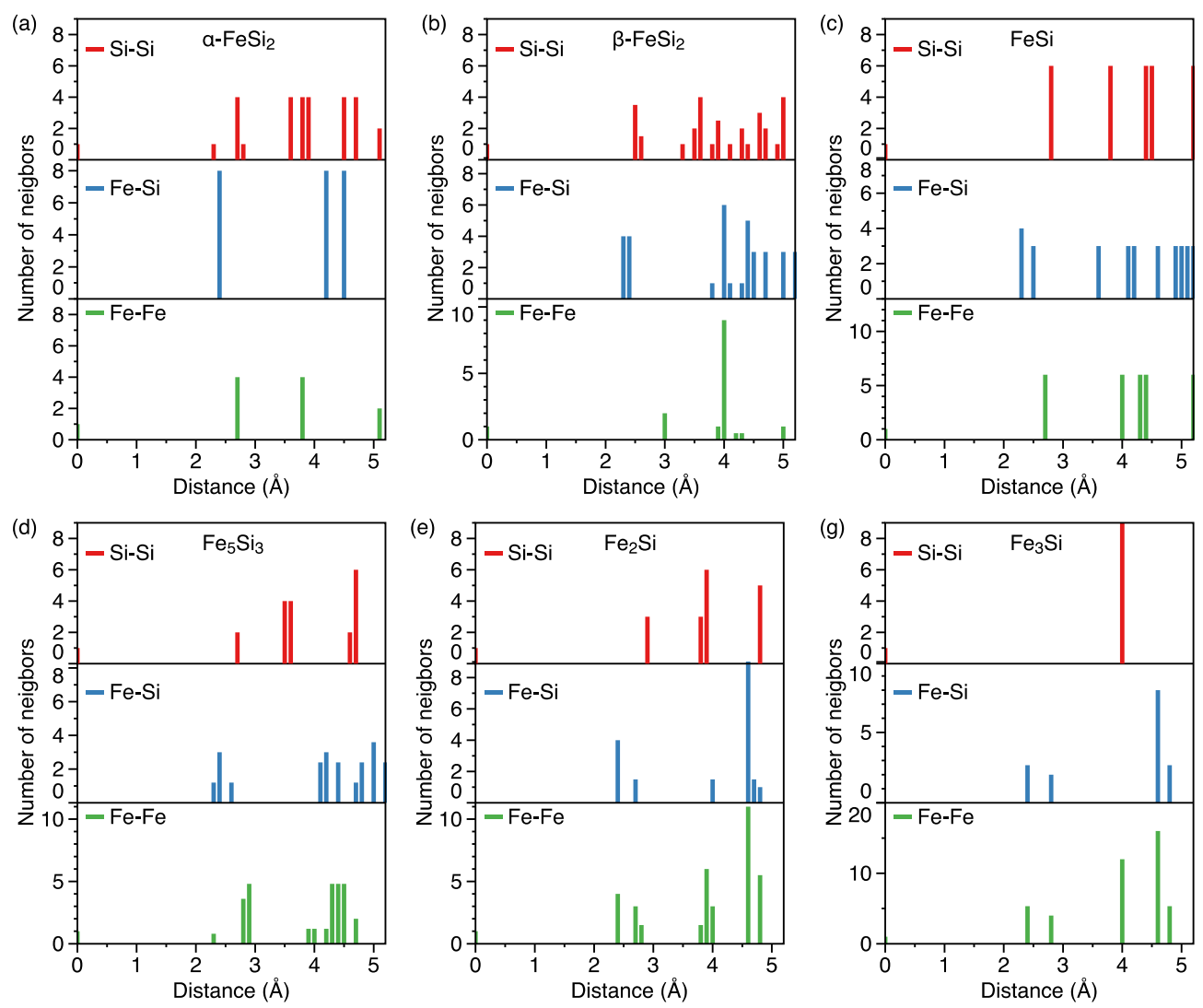

Figure S2. The average number of neighbors at different distances in the unit cell 
Table S5. Thermal contribution of the ideal gas molecule and the ideal gas molecule entropy at different temperature $(\mathrm{T}, \mathrm{K})$ and $1 \mathrm{~atm}$ pressure

$\tilde{\mu}_{i}\left(T, p^{\theta}\right)=\left[H\left(T, p^{\theta}\right)-H^{\theta}\left(T_{r}\right)\right]-T\left[S^{\theta}(T)-S^{\theta}(0 K)\right]$

Enthalpy reference temperature $=T_{\mathrm{r}}=298.15 \mathrm{~K}$

Standard state pressure $=p^{\theta}=0.1 \mathrm{MPa}$

\begin{tabular}{|c|c|c|c|c|}
\hline & \multicolumn{2}{|c|}{$\mathrm{SiH}_{4}$} & \multicolumn{2}{|r|}{$\mathrm{H}_{2}$} \\
\hline $\mathrm{T} / \mathrm{K}$ & $S^{\theta}\left(\mathrm{J} \cdot \mathrm{K}^{-1} \cdot \mathrm{mol}^{-1}\right)$ & $H-H^{\theta}\left(T_{\mathrm{r}}\right)\left(\mathrm{kJ} \cdot \mathrm{mol}^{-1}\right)$ & $S^{\theta}\left(\mathrm{J} \cdot \mathrm{K}^{-1} \cdot \mathrm{mol}^{-1}\right)$ & $H-H^{\theta}\left(T_{\mathrm{r}}\right)\left(\mathrm{kJ} \cdot \mathrm{mol}^{-1}\right)$ \\
\hline 0 & 0 & -10.538 & 0 & -8.467 \\
\hline 100 & 165.774 & -7.212 & 100.727 & -5.468 \\
\hline 200 & 189.213 & -3.819 & 119.412 & -2.774 \\
\hline 250 & 197.475 & -1.965 & 125.64 & -1.378 \\
\hline 298.15 & 204.653 & 0 & 130.68 & 0 \\
\hline 300 & 204.918 & 0.079 & 130.858 & 0.053 \\
\hline 350 & 211.868 & 2.336 & 135.325 & 1.502 \\
\hline 400 & 218.458 & 4.806 & 139.216 & 2.959 \\
\hline 450 & 224.752 & 7.48 & 142.656 & 4.42 \\
\hline 500 & 230.786 & 10.345 & 145.737 & 5.882 \\
\hline 600 & 242.179 & 16.604 & 151.077 & 8.811 \\
\hline 700 & 252.784 & 23.491 & 155.606 & 11.749 \\
\hline 800 & 262.695 & 30.919 & 159.548 & 14.702 \\
\hline 900 & 271.982 & 38.808 & 163.051 & 17.676 \\
\hline 1000 & 280.701 & 47.086 & 166.216 & 20.68 \\
\hline 1100 & 288.9 & 55.692 & 169.112 & 23.719 \\
\hline 1200 & 296.625 & 64.572 & 171.79 & 26.797 \\
\hline 1300 & 303.917 & 73.683 & 174.288 & 29.918 \\
\hline 1400 & 310.814 & 82.99 & 176.633 & 33.082 \\
\hline 1500 & 317.348 & 92.463 & 178.846 & 36.29 \\
\hline
\end{tabular}


Table S6. The value of $\mu$ si under the $\mathrm{p}=1 \mathrm{~atm}, \mathrm{~T}=450 \sim 1500 \mathrm{~K}$, and $\mathrm{a}\left(\mathrm{SiH}_{4} / \mathrm{H}_{2}\right)=$ $1 / 100 \sim 20 / 100$ conditions.

\begin{tabular}{|c|c|c|c|c|c|}
\hline \multirow{2}{*}{$\mathrm{T}(\mathrm{K})$} & \multicolumn{5}{|c|}{$\mu_{\mathrm{Si}}(\mathrm{eV})$} \\
\hline & $\mathrm{a}=1 / 100$ & $\mathrm{a}=5 / 100$ & $\mathrm{a}=10 / 100$ & $\mathrm{a}=15 / 100$ & $\mathrm{a}=20 / 100$ \\
\hline 450 & -5.30 & -5.23 & -5.20 & -5.19 & -5.17 \\
\hline 500 & -5.29 & -5.21 & -5.18 & -5.16 & -5.15 \\
\hline 600 & -5.26 & -5.18 & -5.14 & -5.12 & -5.10 \\
\hline 700 & -5.24 & -5.14 & -5.10 & -5.07 & -5.05 \\
\hline 800 & -5.22 & -5.11 & -5.06 & -5.03 & -5.00 \\
\hline 900 & -5.20 & -5.08 & -5.02 & -4.98 & -4.96 \\
\hline 1000 & -5.19 & -5.05 & -4.98 & -4.94 & -4.91 \\
\hline 1100 & -5.18 & -5.02 & -4.95 & -4.91 & -4.87 \\
\hline 1200 & -5.16 & -4.99 & -4.92 & -4.87 & -4.84 \\
\hline 1300 & -5.16 & -4.97 & -4.89 & -4.84 & -4.80 \\
\hline 1400 & -5.15 & -4.95 & -4.86 & -4.81 & -4.77 \\
\hline 1500 & -5.15 & -4.93 & -4.84 & -4.78 & -4.74 \\
\hline
\end{tabular}

Table S7. Calculated surface energies $\left(\mathrm{eV} / \AA^{2}\right)$ for surfaces of $\mathrm{Fe}_{3} \mathrm{Si}$

\begin{tabular}{c|c|c|c|c|c|c|c}
\hline \multirow{2}{*}{$\mathrm{Fe}_{3} \mathrm{Si}$ surface } & \multicolumn{7}{|c}{ Si potential (eV) } \\
\cline { 2 - 8 } & -6.6 & -6.4 & -6.2 & -6 & -5.8 & -5.6 & -5.4 \\
\hline (100)-Fe1 & 0.180 & 0.184 & 0.189 & 0.193 & 0.197 & 0.202 & 0.206 \\
$(100)-\mathrm{Si} 1$ & 0.133 & 0.128 & 0.124 & 0.120 & 0.115 & 0.111 & 0.107 \\
\hline (110)-SiFe1 & 0.142 & 0.142 & 0.142 & 0.142 & 0.142 & 0.142 & 0.142 \\
\hline (111)-Fe2 & 0.192 & 0.199 & 0.207 & 0.214 & 0.222 & 0.229 & 0.236 \\
(111)-Fe1 & 0.178 & 0.180 & 0.182 & 0.185 & 0.187 & 0.190 & 0.192 \\
(111)-SiFe1 & 0.165 & 0.165 & 0.165 & 0.165 & 0.165 & 0.165 & 0.165 \\
(111)-Si1 & 0.130 & 0.123 & 0.115 & 0.108 & 0.101 & 0.093 & 0.086 \\
\hline (211)-SiFe1 & 0.153 & 0.153 & 0.153 & 0.153 & 0.153 & 0.153 & 0.153 \\
\hline (311)-Si2 & 0.142 & 0.138 & 0.135 & 0.131 & 0.127 & 0.123 & 0.120 \\
(311)-Fe2 & 0.172 & 0.175 & 0.179 & 0.183 & 0.187 & 0.190 & 0.194 \\
(311)-Fe1 & 0.154 & 0.156 & 0.157 & 0.158 & 0.159 & 0.161 & 0.162 \\
(311)-Si1 & 0.170 & 0.169 & 0.168 & 0.166 & 0.165 & 0.164 & 0.163 \\
\hline
\end{tabular}




\begin{tabular}{c|ccccccc}
\hline (310)-SiFe1 & 0.155 & 0.155 & 0.155 & 0.155 & 0.155 & 0.155 & 0.155 \\
\hline
\end{tabular}

Table S8. Calculated surface energies $\left(\mathrm{eV} / \AA^{2}\right)$ for surfaces of $\mathrm{Fe}_{2} \mathrm{Si}$

\begin{tabular}{|c|c|c|c|c|c|c|}
\hline \multirow{2}{*}{$\mathrm{Fe}_{2} \mathrm{Si}$ surface } & \multicolumn{6}{|c|}{ Si potential $(\mathrm{eV})$} \\
\hline & -6.4 & -6.2 & -6 & -5.8 & -5.6 & -5.4 \\
\hline (001)-Fe1 & 0.143 & 0.147 & 0.151 & 0.155 & 0.158 & 0.162 \\
\hline$(001)-\mathrm{Si} 3$ & 0.146 & 0.135 & 0.123 & 0.112 & 0.101 & 0.090 \\
\hline$(001)-\mathrm{Fe} 2$ & 0.259 & 0.270 & 0.282 & 0.293 & 0.304 & 0.315 \\
\hline$(001)-\mathrm{Si} 2$ & 0.122 & 0.118 & 0.114 & 0.111 & 0.107 & 0.103 \\
\hline$(001)-S i 1$ & 0.150 & 0.146 & 0.142 & 0.138 & 0.134 & 0.131 \\
\hline (011)-Fe1 & 0.092 & 0.095 & 0.099 & 0.102 & 0.105 & 0.108 \\
\hline$(011)-\mathrm{Si} 2$ & 0.322 & 0.318 & 0.313 & 0.309 & 0.305 & 0.300 \\
\hline (011)-Fe3 & 0.181 & 0.183 & 0.185 & 0.187 & 0.189 & 0.192 \\
\hline (011)-Si1 & 0.178 & 0.176 & 0.174 & 0.172 & 0.169 & 0.167 \\
\hline (011)-Si3 & 0.148 & 0.141 & 0.135 & 0.128 & 0.122 & 0.115 \\
\hline$(011)-\mathrm{Fe} 2$ & 0.159 & 0.161 & 0.164 & 0.166 & 0.168 & 0.170 \\
\hline$(21-2)-\mathrm{Si} 1$ & 0.162 & 0.160 & 0.158 & 0.157 & 0.155 & 0.153 \\
\hline$(21-2)-\mathrm{Fe} 2$ & 0.168 & 0.170 & 0.171 & 0.173 & 0.175 & 0.177 \\
\hline$(21-2)-\mathrm{SiFe} 1$ & 0.158 & 0.158 & 0.158 & 0.158 & 0.158 & 0.158 \\
\hline$(21-2)-\mathrm{Fe} 1$ & 0.166 & 0.168 & 0.169 & 0.171 & 0.173 & 0.175 \\
\hline$(21-2)-\mathrm{Si} 2$ & 0.155 & 0.153 & 0.151 & 0.149 & 0.147 & 0.146 \\
\hline$(100)-S i 1$ & 0.145 & 0.142 & 0.139 & 0.137 & 0.134 & 0.131 \\
\hline$(100)-\mathrm{Fe} 1$ & 0.169 & 0.174 & 0.179 & 0.184 & 0.190 & 0.195 \\
\hline (101)-Si3 & 0.119 & 0.112 & 0.106 & 0.099 & 0.093 & 0.087 \\
\hline (101)-Si1 & 0.168 & 0.165 & 0.163 & 0.161 & 0.159 & 0.157 \\
\hline$(101)-\mathrm{Fe} 2$ & 0.158 & 0.160 & 0.162 & 0.164 & 0.166 & 0.169 \\
\hline (101)-Si2 & 0.144 & 0.142 & 0.140 & 0.138 & 0.136 & 0.134 \\
\hline (101)-Fe1 & 0.154 & 0.156 & 0.159 & 0.161 & 0.163 & 0.165 \\
\hline (101)-Fe3 & 0.177 & 0.183 & 0.190 & 0.196 & 0.203 & 0.209 \\
\hline (104)-Si3 & 0.266 & 0.259 & 0.252 & 0.245 & 0.238 & 0.231 \\
\hline (104)-SiFe1 & 0.155 & 0.155 & 0.155 & 0.155 & 0.155 & 0.155 \\
\hline (104)-Si2 & 0.130 & 0.129 & 0.127 & 0.125 & 0.123 & 0.122 \\
\hline$(104)-\mathrm{Fe} 2$ & 0.086 & 0.088 & 0.089 & 0.091 & 0.093 & 0.095 \\
\hline (104)-Si1 & 0.137 & 0.136 & 0.135 & 0.134 & 0.133 & 0.132 \\
\hline (104)-Fe1 & 0.140 & 0.142 & 0.144 & 0.146 & 0.147 & 0.149 \\
\hline (102)-Si2 & 0.178 & 0.175 & 0.172 & 0.169 & 0.166 & 0.163 \\
\hline$(102)-\mathrm{Fe} 2$ & 0.179 & 0.185 & 0.191 & 0.197 & 0.203 & 0.209 \\
\hline (102)-Si1 & 0.188 & 0.191 & 0.194 & 0.197 & 0.200 & 0.203 \\
\hline (102)-SiFe1 & 0.135 & 0.135 & 0.135 & 0.135 & 0.135 & 0.135 \\
\hline (102)-Si3 & 0.134 & 0.127 & 0.121 & 0.115 & 0.109 & 0.103 \\
\hline (110)-SiFe1 & 0.136 & 0.136 & 0.136 & 0.136 & 0.136 & 0.136 \\
\hline (111)-Si3 & 0.137 & 0.133 & 0.129 & 0.125 & 0.120 & 0.116 \\
\hline
\end{tabular}




\begin{tabular}{l|llllll} 
(111)-Fe3 & 0.170 & 0.174 & 0.178 & 0.183 & 0.187 & 0.191 \\
(111)-Si1 & 0.151 & 0.150 & 0.149 & 0.147 & 0.146 & 0.144 \\
(111)-Si2 & 0.153 & 0.151 & 0.150 & 0.148 & 0.147 & 0.145 \\
(111)-Fe1 & 0.157 & 0.158 & 0.160 & 0.161 & 0.162 & 0.164 \\
(111)-Fe2 & 0.158 & 0.160 & 0.161 & 0.162 & 0.164 & 0.165 \\
\hline
\end{tabular}

Table S9. Calculated surface energies $\left(\mathrm{eV} / \AA^{2}\right)$ for surfaces of $\mathrm{Fe}_{5} \mathrm{Si}_{3}$

\begin{tabular}{c|c|c|c|c|c|c}
\hline \multirow{2}{*}{ Fesi3 3 surface } & \multicolumn{5}{|c}{ Si potential (eV) } \\
\cline { 2 - 7 } (001)-Fe1 & -6.4 & -6.2 & -6 & -5.8 & -5.6 & -5.4 \\
\hline (001)-Si1 & 0.097 & 0.142 & 0.145 & 0.148 & 0.151 & 0.154 \\
(01-2)-Fe1 & 0.140 & 0.141 & 0.142 & 0.143 & 0.144 & 0.145 \\
(01-2)-Si2 & 0.125 & 0.123 & 0.121 & 0.119 & 0.117 & 0.115 \\
(01-2)-Fe2 & 0.145 & 0.147 & 0.149 & 0.151 & 0.153 & 0.155 \\
(01-2)-Si1 & 0.129 & 0.128 & 0.127 & 0.126 & 0.125 & 0.125 \\
(01-2)-Si3 & 0.121 & 0.116 & 0.112 & 0.107 & 0.102 & 0.097 \\
(01-2)-SiFe1 & 0.117 & 0.117 & 0.117 & 0.117 & 0.117 & 0.117 \\
(01-2)-Fe3 & 0.133 & 0.138 & 0.143 & 0.148 & 0.152 & 0.157 \\
\hline (1-2-2)-Fe1 & 0.129 & 0.129 & 0.130 & 0.130 & 0.130 & 0.131 \\
(1-2-2)-Fe2 & 0.133 & 0.135 & 0.137 & 0.139 & 0.141 & 0.143 \\
(1-2-2)-SiFe1 & 0.124 & 0.124 & 0.124 & 0.124 & 0.124 & 0.124 \\
(1-2-2)-Si1 & 0.132 & 0.131 & 0.131 & 0.130 & 0.130 & 0.129 \\
(1-2-2)-Fe4 & 0.154 & 0.157 & 0.161 & 0.165 & 0.169 & 0.173 \\
(1-2-2)-Fe3 & 0.150 & 0.152 & 0.155 & 0.157 & 0.160 & 0.162 \\
(1-2-2)-Si3 & 0.133 & 0.130 & 0.128 & 0.125 & 0.123 & 0.120 \\
(1-2-2)-Si2 & 0.127 & 0.125 & 0.122 & 0.120 & 0.118 & 0.116 \\
\hline (2-1-1)-Fe4 & 0.141 & 0.147 & 0.152 & 0.158 & 0.163 & 0.168 \\
(2-1-1)-Si4 & 0.132 & 0.127 & 0.121 & 0.116 & 0.111 & 0.105 \\
(2-1-1)-Fe5 & 0.150 & 0.157 & 0.164 & 0.172 & 0.179 & 0.186 \\
(2-1-1)-Si1 & 0.136 & 0.135 & 0.134 & 0.133 & 0.131 & 0.130 \\
(2-1-1)-Si2 & 0.149 & 0.147 & 0.145 & 0.143 & 0.142 & 0.140 \\
(2-1-1)-Fe2 & 0.138 & 0.140 & 0.142 & 0.143 & 0.145 & 0.147 \\
(2-1-1)-Si3 & 0.133 & 0.131 & 0.129 & 0.127 & 0.126 & 0.124 \\
(2-1-1)-Si5 & 0.145 & 0.137 & 0.130 & 0.123 & 0.116 & 0.109 \\
(2-1-1)-Fe1 & 0.145 & 0.146 & 0.148 & 0.149 & 0.150 & 0.151 \\
(2-1-1)-SiFe1 & 0.138 & 0.138 & 0.138 & 0.138 & 0.138 & 0.138 \\
(2-1-1)-Fe3 & 0.144 & 0.146 & 0.148 & 0.150 & 0.151 & 0.153 \\
\hline (3-1-3)-Fe2 & 0.133 & 0.134 & 0.135 & 0.136 & 0.137 & 0.138 \\
(3-1-3)-Fe3 & 0.137 & 0.138 & 0.139 & 0.140 & 0.141 & 0.142 \\
(3-1-3)-Si1 & 0.129 & 0.128 & 0.127 & 0.126 & 0.125 & 0.124 \\
(3-1-3)-Si3 & 0.130 & 0.129 & 0.127 & 0.125 & 0.124 & 0.122 \\
(3-1-3)-Si2 & 0.125 & 0.127 & 0.128 & 0.129 & 0.130 & 0.132 \\
& & & & & & 0.124 \\
\hline
\end{tabular}




\begin{tabular}{|c|c|c|c|c|c|c|}
\hline (3-1-3)-SiFe1 & 0.126 & 0.126 & 0.126 & 0.126 & 0.126 & 0.126 \\
\hline$(3-11)-\mathrm{Fe} 5$ & 0.140 & 0.142 & 0.144 & 0.145 & 0.147 & 0.149 \\
\hline$(3-11)-\mathrm{Fe} 4$ & 0.073 & 0.074 & 0.075 & 0.076 & 0.077 & 0.078 \\
\hline$(3-11)-\mathrm{Fe} 2$ & 0.075 & 0.076 & 0.076 & 0.076 & 0.077 & 0.077 \\
\hline$(3-11)-\mathrm{Fe} 3$ & 0.137 & 0.137 & 0.138 & 0.138 & 0.138 & 0.139 \\
\hline$(3-11)-\mathrm{Si} 3$ & 0.134 & 0.133 & 0.132 & 0.131 & 0.130 & 0.129 \\
\hline (3-11)-Si5 & 0.075 & 0.074 & 0.072 & 0.071 & 0.070 & 0.068 \\
\hline (3-11)-Si1 & 0.149 & 0.149 & 0.148 & 0.148 & 0.147 & 0.147 \\
\hline (3-11)-SiFe1 & 0.074 & 0.074 & 0.074 & 0.074 & 0.074 & 0.074 \\
\hline$(3-11)-\mathrm{Fe} 1$ & 0.069 & 0.069 & 0.070 & 0.070 & 0.070 & 0.071 \\
\hline$(3-11)-S i 2$ & 0.074 & 0.073 & 0.072 & 0.072 & 0.071 & 0.070 \\
\hline$(3-11)-\mathrm{SiFe} 2$ & 0.068 & 0.068 & 0.068 & 0.068 & 0.068 & 0.068 \\
\hline$(3-11)-\mathrm{Si} 4$ & 0.069 & 0.068 & 0.067 & 0.065 & 0.064 & 0.063 \\
\hline (011)-SiFe1 & 0.128 & 0.128 & 0.128 & 0.128 & 0.128 & 0.128 \\
\hline (011)-Si1 & 0.138 & 0.138 & 0.137 & 0.136 & 0.135 & 0.134 \\
\hline (011)-Fe1 & 0.134 & 0.135 & 0.136 & 0.137 & 0.138 & 0.138 \\
\hline$(011)-\mathrm{Fe} 2$ & 0.143 & 0.145 & 0.147 & 0.148 & 0.150 & 0.151 \\
\hline (011)-Si3 & 0.136 & 0.133 & 0.131 & 0.128 & 0.126 & 0.123 \\
\hline$(011)-\mathrm{Si} 2$ & 0.127 & 0.125 & 0.124 & 0.122 & 0.120 & 0.119 \\
\hline$(011)-\mathrm{Fe} 3$ & 0.128 & 0.130 & 0.133 & 0.135 & 0.137 & 0.140 \\
\hline$(100)-F e 1$ & 0.134 & 0.137 & 0.139 & 0.142 & 0.144 & 0.147 \\
\hline$(100)-\mathrm{Fe} 2$ & 0.183 & 0.188 & 0.193 & 0.199 & 0.204 & 0.209 \\
\hline (100)-Si1 & 0.142 & 0.140 & 0.137 & 0.135 & 0.132 & 0.130 \\
\hline$(100)-\mathrm{SiFe} 1$ & 0.136 & 0.136 & 0.136 & 0.136 & 0.136 & 0.136 \\
\hline (100)-Si2 & 0.158 & 0.153 & 0.148 & 0.142 & 0.137 & 0.132 \\
\hline$(100)-\mathrm{Fe} 3$ & 0.188 & 0.201 & 0.214 & 0.227 & 0.239 & 0.252 \\
\hline$(110)-\mathrm{Fe} 5$ & 0.168 & 0.173 & 0.178 & 0.184 & 0.189 & 0.194 \\
\hline (110)-Fe2 & 0.140 & 0.142 & 0.144 & 0.147 & 0.149 & 0.151 \\
\hline (110)-SiFe1 & 0.130 & 0.130 & 0.130 & 0.130 & 0.130 & 0.130 \\
\hline (110)-Fe1 & 0.145 & 0.146 & 0.147 & 0.147 & 0.148 & 0.149 \\
\hline$(110)-\mathrm{Fe} 3$ & 0.145 & 0.140 & 0.136 & 0.131 & 0.127 & 0.122 \\
\hline (110)-Si1 & 0.131 & 0.130 & 0.130 & 0.129 & 0.128 & 0.127 \\
\hline (110)-Si3 & 0.136 & 0.131 & 0.126 & 0.120 & 0.115 & 0.110 \\
\hline (110)-Si2 & 0.160 & 0.158 & 0.155 & 0.153 & 0.151 & 0.149 \\
\hline (110)-Fe4 & 0.158 & 0.162 & 0.167 & 0.171 & 0.175 & 0.180 \\
\hline$(120)-F e 6$ & 0.156 & 0.160 & 0.163 & 0.167 & 0.170 & 0.173 \\
\hline$(120)-\mathrm{Fe} 1$ & 0.155 & 0.154 & 0.153 & 0.152 & 0.151 & 0.150 \\
\hline$(120)-\mathrm{SiFe} 1$ & 0.161 & 0.161 & 0.161 & 0.161 & 0.161 & 0.161 \\
\hline$(120)-\mathrm{SiFe} 2$ & 0.157 & 0.157 & 0.157 & 0.157 & 0.157 & 0.157 \\
\hline$(120)-\mathrm{Si} 2$ & 0.156 & 0.154 & 0.153 & 0.151 & 0.149 & 0.147 \\
\hline (120)-Si1 & 0.159 & 0.156 & 0.153 & 0.149 & 0.146 & 0.142 \\
\hline$(120)-\mathrm{Fe} 5$ & 0.142 & 0.144 & 0.147 & 0.149 & 0.151 & 0.154 \\
\hline$(120)-\mathrm{Fe} 7$ & 0.158 & 0.162 & 0.167 & 0.172 & 0.177 & 0.182 \\
\hline$(120)-\mathrm{Fe} 4$ & 0.149 & 0.151 & 0.153 & 0.155 & 0.157 & 0.159 \\
\hline
\end{tabular}




\begin{tabular}{c|cccccc} 
(120)-Fe3 & 0.156 & 0.157 & 0.159 & 0.160 & 0.162 & 0.163 \\
(120)-SiFe3 & 0.133 & 0.133 & 0.133 & 0.133 & 0.133 & 0.133 \\
(120)-Fe2 & 0.167 & 0.168 & 0.169 & 0.170 & 0.171 & 0.172 \\
\hline (231)-SiFe1 & 0.140 & 0.140 & 0.140 & 0.140 & 0.140 & 0.140 \\
(231)-Fe4 & 0.141 & 0.143 & 0.144 & 0.145 & 0.147 & 0.148 \\
(231)-Fe3 & 0.139 & 0.141 & 0.142 & 0.143 & 0.144 & 0.145 \\
(231)-Fe2 & 0.140 & 0.141 & 0.142 & 0.143 & 0.144 & 0.145 \\
(231)-Si2 & 0.136 & 0.135 & 0.134 & 0.134 & 0.133 & 0.132 \\
(231)-Si1 & 0.143 & 0.142 & 0.142 & 0.142 & 0.141 & 0.141 \\
(231)-Fe1 & 0.138 & 0.139 & 0.139 & 0.139 & 0.140 & 0.140 \\
\hline
\end{tabular}

Table S10. Calculated surface energies $\left(\mathrm{eV} / \AA^{2}\right)$ for surfaces of FeSi

\begin{tabular}{c|c|c|c|c|c|c}
\hline \multirow{2}{*}{ FeSi surface } & \multicolumn{5}{|c}{ Si potential (eV) } \\
\cline { 2 - 7 } & -6.4 & -6.2 & -6 & -5.8 & -5.6 & -5.4 \\
\hline (100)-Fe1 & 0.185 & 0.195 & 0.205 & 0.215 & 0.225 & 0.236 \\
(100)-Si1 & 0.148 & 0.138 & 0.128 & 0.117 & 0.107 & 0.097 \\
(100)-SiFe1 & 0.167 & 0.167 & 0.167 & 0.167 & 0.167 & 0.167 \\
(100)-SiFe2 & 0.138 & 0.138 & 0.138 & 0.138 & 0.138 & 0.138 \\
\hline (110)-Fe2 & 0.183 & 0.190 & 0.197 & 0.205 & 0.212 & 0.219 \\
(110)-SiFe2 & 0.169 & 0.169 & 0.169 & 0.169 & 0.169 & 0.169 \\
(110)-Si2 & 0.150 & 0.143 & 0.136 & 0.129 & 0.122 & 0.115 \\
(110)-SiFe1 & 0.140 & 0.140 & 0.140 & 0.140 & 0.140 & 0.140 \\
(110)-Si1 & 0.155 & 0.147 & 0.140 & 0.133 & 0.126 & 0.119 \\
(110)-Fe1 & 0.147 & 0.154 & 0.161 & 0.168 & 0.176 & 0.183 \\
\hline (111)-Fe2 & 0.178 & 0.178 & 0.178 & 0.178 & 0.178 & 0.178 \\
(111)-Fe1 & 0.150 & 0.150 & 0.150 & 0.150 & 0.150 & 0.150 \\
(111)-Si1 & 0.156 & 0.156 & 0.156 & 0.156 & 0.156 & 0.156 \\
(111)-Si2 & 0.147 & 0.147 & 0.147 & 0.147 & 0.147 & 0.147 \\
\hline (120)-Fe3 & 0.166 & 0.175 & 0.184 & 0.193 & 0.202 & 0.211 \\
(120)-Fe2 & 0.163 & 0.168 & 0.172 & 0.177 & 0.181 & 0.186 \\
(120)-SiFe2 & 0.159 & 0.159 & 0.159 & 0.159 & 0.159 & 0.159 \\
(120)-Si1 & 0.158 & 0.153 & 0.148 & 0.144 & 0.139 & 0.135 \\
(120)-Si3 & 0.151 & 0.142 & 0.133 & 0.124 & 0.115 & 0.106 \\
(120)-Si2 & 0.140 & 0.135 & 0.131 & 0.126 & 0.122 & 0.117 \\
(120)-SiFe1 & 0.164 & 0.164 & 0.164 & 0.164 & 0.164 & 0.164 \\
(120)-Fe1 & 0.170 & 0.175 & 0.179 & 0.184 & 0.188 & 0.193 \\
\hline (210)-Fe1 & 0.172 & 0.177 & 0.181 & 0.186 & 0.191 & 0.195 \\
(210)-SiFe1 & 0.177 & 0.177 & 0.177 & 0.177 & 0.177 & 0.177 \\
(210)-SiFe2 & 0.163 & 0.163 & 0.163 & 0.163 & 0.163 & 0.163 \\
(210)-Si2 & 0.153 & 0.149 & 0.144 & 0.140 & 0.135 & 0.131 \\
(210)-Si1 & 0.177 & 0.172 & 0.168 & 0.163 & 0.159 & 0.154 \\
(210)-SiFe3 & 0.186 & 0.186 & 0.186 & 0.186 & 0.186 & 0.186 \\
(210)-SiFe4 & 0.140 & 0.140 & 0.140 & 0.140 & 0.140 & 0.140 \\
& & & & & &
\end{tabular}




\begin{tabular}{|c|c|c|c|c|c|c|}
\hline (210)-Fe2 & 0.176 & 0.180 & 0.185 & 0.190 & 0.194 & 0.199 \\
\hline (211)-Si2 & 0.159 & 0.159 & 0.159 & 0.159 & 0.159 & 0.159 \\
\hline (211)-Fe3 & 0.163 & 0.163 & 0.163 & 0.163 & 0.163 & 0.163 \\
\hline (211)-Fe2 & 0.156 & 0.156 & 0.156 & 0.156 & 0.156 & 0.156 \\
\hline (211)-Fe1 & 0.156 & 0.156 & 0.156 & 0.156 & 0.156 & 0.156 \\
\hline (211)-SiFe1 & 0.158 & 0.158 & 0.158 & 0.158 & 0.158 & 0.158 \\
\hline (211)-Si3 & 0.154 & 0.154 & 0.154 & 0.154 & 0.154 & 0.154 \\
\hline (211)-Si1 & 0.166 & 0.166 & 0.166 & 0.166 & 0.166 & 0.166 \\
\hline (311)-Si1 & 0.152 & 0.152 & 0.152 & 0.152 & 0.152 & 0.152 \\
\hline (311)-Fe1 & 0.158 & 0.158 & 0.158 & 0.158 & 0.158 & 0.158 \\
\hline (311)-Fe2 & 0.156 & 0.156 & 0.156 & 0.156 & 0.156 & 0.156 \\
\hline$(311)-\mathrm{Fe} 3$ & 0.158 & 0.158 & 0.158 & 0.158 & 0.158 & 0.158 \\
\hline (311)-SiFe1 & 0.142 & 0.142 & 0.142 & 0.142 & 0.142 & 0.142 \\
\hline (311)-Si2 & 0.160 & 0.160 & 0.160 & 0.160 & 0.160 & 0.160 \\
\hline (123)-Si1 & 0.158 & 0.158 & 0.158 & 0.158 & 0.158 & 0.158 \\
\hline (123)-SiFe1 & 0.158 & 0.158 & 0.158 & 0.158 & 0.158 & 0.158 \\
\hline (123)-Fe1 & 0.164 & 0.164 & 0.164 & 0.164 & 0.164 & 0.164 \\
\hline (123)-Fe2 & 0.167 & 0.167 & 0.167 & 0.167 & 0.167 & 0.167 \\
\hline (123)-Si2 & 0.162 & 0.162 & 0.162 & 0.162 & 0.162 & 0.162 \\
\hline (123)-Si3 & 0.156 & 0.156 & 0.156 & 0.156 & 0.156 & 0.156 \\
\hline (123)-Si4 & 0.152 & 0.152 & 0.152 & 0.152 & 0.152 & 0.152 \\
\hline
\end{tabular}

Table S11. Calculated surface energies $\left(e V / \AA^{2}\right)$ for surfaces of $\alpha-\mathrm{FeSi}_{2}$

\begin{tabular}{c|c|c|c|c}
\hline \multirow{2}{*}{$\alpha-\mathrm{FeSi}$ surface } & \multicolumn{4}{|c}{ Si potential (eV) } \\
\cline { 2 - 5 } & -6 & -5.8 & -5.6 & -5.4 \\
\hline$(001)-\mathrm{Fe} 1$ & 0.199 & 0.227 & 0.254 & 0.282 \\
$(001)-\mathrm{SiFe} 1$ & 0.130 & 0.130 & 0.130 & 0.130 \\
$(001)-\mathrm{Si} 1$ & 0.160 & 0.132 & 0.105 & 0.077 \\
\hline (10-2)-Fe1 & 0.149 & 0.159 & 0.169 & 0.179 \\
$(10-2)-\mathrm{SiFe} 1$ & 0.114 & 0.114 & 0.114 & 0.114 \\
$(10-2)-\mathrm{Si} 1$ & 0.120 & 0.110 & 0.100 & 0.090 \\
\hline (101)-Fe1 & 0.170 & 0.183 & 0.196 & 0.208 \\
$(101)-\mathrm{SiFe} 1$ & 0.137 & 0.124 & 0.111 & 0.099 \\
$(101)-\mathrm{Si} 1$ & 0.099 & 0.099 & 0.099 & 0.099 \\
\hline (110)-SiFe1 & 0.103 & 0.103 & 0.103 & 0.103 \\
\hline (111)-Fe1 & 0.127 & 0.137 & 0.147 & 0.156 \\
$(111)-\mathrm{SiFe} 1$ & 0.120 & 0.120 & 0.120 & 0.120 \\
$(111)-\mathrm{Si} 1$ & 0.129 & 0.120 & 0.110 & 0.101 \\
\hline (113)-Fe1 & 0.140 & 0.147 & 0.153 & 0.160 \\
$(113)-\mathrm{SiFe} 1$ & 0.127 & 0.127 & 0.127 & 0.127 \\
$(113)-\mathrm{Si} 1$ & 0.133 & 0.127 & 0.120 & 0.113 \\
\hline (114)-Fe1 & 0.144 & 0.149 & 0.155 & 0.161 \\
& & & &
\end{tabular}




\begin{tabular}{c|llll}
$(114)-\mathrm{SiFe} 1$ & 0.132 & 0.132 & 0.132 & 0.132 \\
$(114)-\mathrm{Si} 1$ & 0.134 & 0.128 & 0.122 & 0.117 \\
\hline (122)-Fe1 & 0.123 & 0.129 & 0.135 & 0.141 \\
$(122)-\mathrm{SiFe} 1$ & 0.122 & 0.122 & 0.122 & 0.122 \\
$(122)-\mathrm{Si} 1$ & 0.117 & 0.111 & 0.105 & 0.099 \\
\hline$(100)-\mathrm{Fe} 1$ & 0.145 & 0.160 & 0.174 & 0.189 \\
$(100)-\mathrm{Si1}$ & 0.166 & 0.152 & 0.138 & 0.123 \\
\hline
\end{tabular}

Table S12. Calculated surface energies $\left(\mathrm{eV} / \AA^{2}\right)$ for surfaces of $\beta-\mathrm{FeSi} 2$

\begin{tabular}{|c|c|c|c|c|}
\hline \multirow{2}{*}{$\beta-\mathrm{FeSi}_{2}$ surface } & \multicolumn{4}{|c|}{ Si potential $(\mathrm{eV})$} \\
\hline & -6 & -5.8 & -5.6 & -5.4 \\
\hline (001)-SiFe3 & 0.102 & 0.102 & 0.102 & 0.102 \\
\hline$(001)-F e 1$ & 0.069 & 0.079 & 0.090 & 0.100 \\
\hline$(001)-\mathrm{Fe} 2$ & 0.142 & 0.152 & 0.162 & 0.173 \\
\hline (001)-SiFe1 & 0.143 & 0.143 & 0.143 & 0.143 \\
\hline (001)-SiFe2 & 0.099 & 0.099 & 0.099 & 0.099 \\
\hline (001)-Si1 & 0.130 & 0.119 & 0.109 & 0.098 \\
\hline$(001)-\mathrm{Si} 2$ & 0.127 & 0.117 & 0.106 & 0.096 \\
\hline (010)-SiFe 1 & 0.142 & 0.142 & 0.142 & 0.142 \\
\hline$(010)-\mathrm{Fe} 1$ & 0.135 & 0.145 & 0.156 & 0.166 \\
\hline$(010)-\mathrm{Fe} 2$ & 0.182 & 0.193 & 0.203 & 0.213 \\
\hline (010)-Si2 & 0.168 & 0.158 & 0.148 & 0.137 \\
\hline (010)-Si1 & 0.175 & 0.164 & 0.154 & 0.143 \\
\hline (010)-SiFe2 & 0.139 & 0.139 & 0.139 & 0.139 \\
\hline (010)-SiFe3 & 0.167 & 0.167 & 0.167 & 0.167 \\
\hline (011)-Fe5 & 0.175 & 0.190 & 0.204 & 0.219 \\
\hline (011)-Fe4 & 0.170 & 0.181 & 0.192 & 0.203 \\
\hline (011)-Si2 & 0.161 & 0.153 & 0.146 & 0.138 \\
\hline$(011)-\mathrm{Fe} 3$ & 0.167 & 0.175 & 0.182 & 0.189 \\
\hline (011)-Si5 & 0.154 & 0.143 & 0.132 & 0.121 \\
\hline (011)-Si4 & 0.158 & 0.147 & 0.136 & 0.125 \\
\hline (011)-Si3 & 0.155 & 0.148 & 0.140 & 0.133 \\
\hline (011)-Si1 & 0.154 & 0.150 & 0.146 & 0.143 \\
\hline (011)-SiFe1 & 0.163 & 0.163 & 0.163 & 0.163 \\
\hline$(011)-\mathrm{Fe} 1$ & 0.164 & 0.168 & 0.172 & 0.175 \\
\hline$(011)-\mathrm{Fe} 2$ & 0.160 & 0.168 & 0.175 & 0.182 \\
\hline (041)-Si2 & 0.143 & 0.140 & 0.137 & 0.135 \\
\hline (041)-Fe1 & 0.156 & 0.158 & 0.161 & 0.163 \\
\hline (041)-Fe3 & 0.143 & 0.145 & 0.148 & 0.150 \\
\hline (041)-Fe4 & 0.150 & 0.153 & 0.157 & 0.161 \\
\hline (041)-SiFe3 & 0.144 & 0.144 & 0.144 & 0.144 \\
\hline$(041)-\mathrm{Si} 3$ & 0.140 & 0.138 & 0.135 & 0.133 \\
\hline (041)-Si4 & 0.138 & 0.136 & 0.133 & 0.131 \\
\hline
\end{tabular}




\begin{tabular}{|c|c|c|c|c|}
\hline (041)-SiFe2 & 0.142 & 0.142 & 0.142 & 0.142 \\
\hline (041)-Si5 & 0.146 & 0.143 & 0.141 & 0.138 \\
\hline (041)-Fe2 & 0.150 & 0.152 & 0.155 & 0.157 \\
\hline (041)-SiFe1 & 0.147 & 0.147 & 0.147 & 0.147 \\
\hline (041)-Si6 & 0.153 & 0.148 & 0.143 & 0.138 \\
\hline (041)-Si1 & 0.153 & 0.151 & 0.148 & 0.146 \\
\hline (111)-SiFe1 & 0.125 & 0.125 & 0.125 & 0.125 \\
\hline (111)-Fe3 & 0.137 & 0.143 & 0.150 & 0.156 \\
\hline (111)-Fe4 & 0.138 & 0.144 & 0.151 & 0.157 \\
\hline (111)-SiFe2 & 0.122 & 0.122 & 0.122 & 0.122 \\
\hline (111)-Fe2 & 0.133 & 0.139 & 0.146 & 0.152 \\
\hline (111)-Fe5 & 0.150 & 0.156 & 0.163 & 0.169 \\
\hline (111)-Si4 & 0.111 & 0.104 & 0.098 & 0.091 \\
\hline (111)-Si6 & 0.106 & 0.099 & 0.093 & 0.087 \\
\hline (111)-SiFe3 & 0.129 & 0.129 & 0.129 & 0.129 \\
\hline (111)-Sil & 0.115 & 0.112 & 0.109 & 0.105 \\
\hline (111)-SiFe4 & 0.121 & 0.121 & 0.121 & 0.121 \\
\hline (111)-Fe1 & 0.132 & 0.135 & 0.138 & 0.141 \\
\hline (111)-Si5 & 0.110 & 0.104 & 0.097 & 0.091 \\
\hline (111)-Si2 & 0.110 & 0.107 & 0.104 & 0.101 \\
\hline (111)-Si3 & 0.112 & 0.106 & 0.099 & 0.093 \\
\hline (111)-SiFe5 & 0.118 & 0.118 & 0.118 & 0.118 \\
\hline (111)-SiFe6 & 0.137 & 0.137 & 0.137 & 0.137 \\
\hline (114)-Fe2 & 0.123 & 0.124 & 0.125 & 0.126 \\
\hline (114)-SiFe1 & 0.123 & 0.123 & 0.123 & 0.123 \\
\hline (114)-Fe3 & 0.121 & 0.123 & 0.124 & 0.125 \\
\hline (114)-Fe7 & 0.118 & 0.121 & 0.123 & 0.126 \\
\hline (114)-Fe8 & 0.120 & 0.124 & 0.128 & 0.131 \\
\hline (114)-Fe4 & 0.117 & 0.118 & 0.119 & 0.120 \\
\hline (114)-Si4 & 0.115 & 0.113 & 0.112 & 0.111 \\
\hline (114)-Fe1 & 0.114 & 0.114 & 0.115 & 0.116 \\
\hline (114)-Si5 & 0.115 & 0.112 & 0.110 & 0.107 \\
\hline (114)-Fe6 & 0.101 & 0.102 & 0.104 & 0.105 \\
\hline (114)-SiFe2 & 0.109 & 0.109 & 0.109 & 0.109 \\
\hline (114)-SiFe3 & 0.105 & 0.105 & 0.105 & 0.105 \\
\hline (114)-Si6 & 0.122 & 0.119 & 0.117 & 0.114 \\
\hline (114)-Si2 & 0.123 & 0.122 & 0.120 & 0.119 \\
\hline (114)-Si7 & 0.113 & 0.109 & 0.105 & 0.102 \\
\hline (114)-Fe5 & 0.107 & 0.108 & 0.109 & 0.110 \\
\hline (114)-Fe6 & 0.108 & 0.110 & 0.113 & 0.115 \\
\hline (114)-Si3 & 0.119 & 0.118 & 0.117 & 0.116 \\
\hline (114)-Si1 & 0.125 & 0.123 & 0.122 & 0.121 \\
\hline (211)-Fe5 & 0.143 & 0.145 & 0.148 & 0.150 \\
\hline (211)-Fe3 & 0.147 & 0.148 & 0.149 & 0.150 \\
\hline
\end{tabular}




\begin{tabular}{|c|c|c|c|c|}
\hline (211)-Si1 & 0.153 & 0.152 & 0.151 & 0.149 \\
\hline (211)-SiFe4 & 0.152 & 0.152 & 0.152 & 0.152 \\
\hline (211)-Si5 & 0.154 & 0.152 & 0.149 & 0.147 \\
\hline (211)-SiFe5 & 0.122 & 0.122 & 0.122 & 0.122 \\
\hline (211)-SiFe6 & 0.130 & 0.130 & 0.130 & 0.130 \\
\hline (211)-Si3 & 0.135 & 0.134 & 0.133 & 0.132 \\
\hline (211)-Si8 & 0.147 & 0.144 & 0.140 & 0.136 \\
\hline (211)-Fe4 & 0.119 & 0.122 & 0.124 & 0.127 \\
\hline (211)-Si6 & 0.146 & 0.144 & 0.141 & 0.139 \\
\hline (211)-Si2 & 0.147 & 0.146 & 0.145 & 0.144 \\
\hline (211)-SiFe3 & 0.152 & 0.152 & 0.152 & 0.152 \\
\hline (211)-Si4 & 0.133 & 0.131 & 0.130 & 0.129 \\
\hline (211)-Si7 & 0.130 & 0.128 & 0.125 & 0.123 \\
\hline (211)-Fe6 & 0.135 & 0.138 & 0.142 & 0.145 \\
\hline (211)-Fe1 & 0.132 & 0.133 & 0.135 & 0.136 \\
\hline (211)-SiFe1 & 0.147 & 0.147 & 0.147 & 0.147 \\
\hline (211)-Fe2 & 0.142 & 0.143 & 0.145 & 0.146 \\
\hline (211)-SiFe2 & 0.133 & 0.133 & 0.133 & 0.133 \\
\hline (131)-SiFe 1 & 0.137 & 0.137 & 0.137 & 0.137 \\
\hline (131)-Si2 & 0.136 & 0.134 & 0.132 & 0.131 \\
\hline (131)-Si3 & 0.136 & 0.135 & 0.133 & 0.132 \\
\hline$(131)-\mathrm{Fe} 2$ & 0.149 & 0.154 & 0.159 & 0.163 \\
\hline (131)-Si1 & 0.140 & 0.139 & 0.137 & 0.135 \\
\hline (131)-Fe3 & 0.135 & 0.139 & 0.144 & 0.149 \\
\hline (131)-Si5 & 0.146 & 0.141 & 0.136 & 0.132 \\
\hline (131)-Si4 & 0.139 & 0.135 & 0.132 & 0.129 \\
\hline (131)-Fe1 & 0.137 & 0.140 & 0.143 & 0.147 \\
\hline (312)-Fe3 & 0.136 & 0.139 & 0.143 & 0.146 \\
\hline (312)-SiFe 1 & 0.126 & 0.126 & 0.126 & 0.126 \\
\hline (312)-Si2 & 0.134 & 0.132 & 0.131 & 0.129 \\
\hline (312)-Fe4 & 0.137 & 0.141 & 0.146 & 0.151 \\
\hline$(312)-\mathrm{Fe} 5$ & 0.145 & 0.150 & 0.155 & 0.160 \\
\hline$(312)-\mathrm{Fe} 2$ & 0.141 & 0.142 & 0.144 & 0.146 \\
\hline (312)-Si1 & 0.137 & 0.135 & 0.134 & 0.132 \\
\hline (312)-SiFe2 & 0.119 & 0.119 & 0.119 & 0.119 \\
\hline (312)-Si4 & 0.142 & 0.137 & 0.132 & 0.127 \\
\hline (312)-Fe1 & 0.132 & 0.134 & 0.136 & 0.137 \\
\hline (312)-Si3 & 0.134 & 0.129 & 0.124 & 0.119 \\
\hline$(421)-\mathrm{Fe} 2$ & 0.125 & 0.128 & 0.131 & 0.134 \\
\hline$(421)-\mathrm{Fe} 1$ & 0.128 & 0.129 & 0.130 & 0.132 \\
\hline$(421)-\mathrm{Fe} 3$ & 0.126 & 0.129 & 0.131 & 0.134 \\
\hline (421)-SiFe1 & 0.130 & 0.130 & 0.130 & 0.130 \\
\hline (421)-SiFe2 & 0.128 & 0.128 & 0.128 & 0.128 \\
\hline (421)-SiFe3 & 0.130 & 0.130 & 0.130 & 0.130 \\
\hline
\end{tabular}




\begin{tabular}{|c|c|c|c|c|}
\hline (421)-Si1 & 0.133 & 0.130 & 0.127 & 0.125 \\
\hline (421)-SiFe4 & 0.126 & 0.126 & 0.126 & 0.126 \\
\hline (421)-SiFe5 & 0.125 & 0.125 & 0.125 & 0.125 \\
\hline$(100)-\mathrm{Fe} 2$ & 0.160 & 0.173 & 0.186 & 0.200 \\
\hline (100)-SiFe1 & 0.145 & 0.145 & 0.145 & 0.145 \\
\hline$(100)-\mathrm{Fe} 1$ & 0.159 & 0.172 & 0.185 & 0.198 \\
\hline$(100)-\mathrm{Si} 2$ & 0.157 & 0.144 & 0.131 & 0.118 \\
\hline (100)-Si1 & 0.139 & 0.125 & 0.112 & 0.099 \\
\hline$(101)-\mathrm{Fe} 5$ & 0.181 & 0.197 & 0.213 & 0.230 \\
\hline (101)-Fe2 & 0.173 & 0.182 & 0.190 & 0.198 \\
\hline (101)-SiFe1 & 0.164 & 0.164 & 0.164 & 0.164 \\
\hline (101)-Si2 & 0.134 & 0.126 & 0.118 & 0.109 \\
\hline$(101)-\mathrm{Si} 5$ & 0.141 & 0.125 & 0.108 & 0.092 \\
\hline (101)-Si4 & 0.145 & 0.132 & 0.120 & 0.108 \\
\hline$(101)-\mathrm{Si} 3$ & 0.129 & 0.121 & 0.113 & 0.105 \\
\hline (101)-Si1 & 0.115 & 0.111 & 0.107 & 0.103 \\
\hline (101)-SiFe2 & 0.108 & 0.108 & 0.108 & 0.108 \\
\hline (101)-Fe1 & 0.136 & 0.140 & 0.144 & 0.148 \\
\hline (101)-Fe3 & 0.157 & 0.165 & 0.173 & 0.181 \\
\hline (101)-Fe4 & 0.169 & 0.181 & 0.193 & 0.206 \\
\hline$(110)-\mathrm{Fe} 2$ & 0.185 & 0.201 & 0.217 & 0.234 \\
\hline (110)-Fe1 & 0.170 & 0.179 & 0.187 & 0.195 \\
\hline (110)-SiFe1 & 0.168 & 0.168 & 0.168 & 0.168 \\
\hline (110)-Si2 & 0.153 & 0.145 & 0.137 & 0.129 \\
\hline (110)-Si5 & 0.165 & 0.148 & 0.132 & 0.116 \\
\hline (110)-Si4 & 0.147 & 0.135 & 0.123 & 0.110 \\
\hline (110)-Si3 & 0.139 & 0.130 & 0.122 & 0.114 \\
\hline (110)-Si1 & 0.126 & 0.122 & 0.117 & 0.113 \\
\hline (110)-SiFe2 & 0.117 & 0.117 & 0.117 & 0.117 \\
\hline$(012)-\mathrm{Fe} 4$ & 0.156 & 0.160 & 0.165 & 0.170 \\
\hline (012)-SiFe1 & 0.153 & 0.153 & 0.153 & 0.153 \\
\hline$(012)-\mathrm{Fe} 2$ & 0.156 & 0.159 & 0.161 & 0.163 \\
\hline (012)-Si2 & 0.151 & 0.148 & 0.146 & 0.144 \\
\hline (012)-SiFe2 & 0.154 & 0.154 & 0.154 & 0.154 \\
\hline (012)-SiFe3 & 0.151 & 0.151 & 0.151 & 0.151 \\
\hline$(012)-\mathrm{Fe} 3$ & 0.155 & 0.157 & 0.160 & 0.162 \\
\hline$(012)-\mathrm{Fe} 1$ & 0.158 & 0.160 & 0.163 & 0.165 \\
\hline$(012)-\mathrm{Fe} 5$ & 0.157 & 0.162 & 0.167 & 0.171 \\
\hline (012)-Si5 & 0.148 & 0.144 & 0.139 & 0.134 \\
\hline$(012)-\mathrm{Si} 3$ & 0.147 & 0.145 & 0.142 & 0.140 \\
\hline (012)-Si1 & 0.152 & 0.150 & 0.148 & 0.145 \\
\hline (012)-Si4 & 0.151 & 0.147 & 0.142 & 0.137 \\
\hline (331)-Fe1 & 0.120 & 0.122 & 0.123 & 0.124 \\
\hline (331)-SiFe1 & 0.124 & 0.124 & 0.124 & 0.124 \\
\hline
\end{tabular}




\begin{tabular}{c|llll} 
(331)-Fe2 & 0.109 & 0.110 & 0.112 & 0.113 \\
(331)-Si1 & 0.123 & 0.122 & 0.120 & 0.119 \\
(331)-Si3 & 0.117 & 0.115 & 0.112 & 0.110 \\
(331)-Si6 & 0.113 & 0.109 & 0.105 & 0.101 \\
(331)-Si4 & 0.113 & 0.111 & 0.108 & 0.106 \\
(331)-Si5 & 0.119 & 0.115 & 0.111 & 0.107 \\
(331)-Fe3 & 0.132 & 0.134 & 0.137 & 0.140 \\
(331)-Si2 & 0.119 & 0.118 & 0.117 & 0.115 \\
\hline (711)-Fe4 & 0.146 & 0.149 & 0.152 & 0.155 \\
(711)-Fe5 & 0.148 & 0.150 & 0.153 & 0.156 \\
(711)-Fe6 & 0.141 & 0.145 & 0.149 & 0.152 \\
(711)-Si6 & 0.143 & 0.140 & 0.136 & 0.132 \\
(711)-Si2 & 0.131 & 0.130 & 0.129 & 0.127 \\
(711)-Si4 & 0.141 & 0.138 & 0.135 & 0.133 \\
(711)-Fe1 & 0.138 & 0.138 & 0.139 & 0.140 \\
(711)-Fe2 & 0.135 & 0.137 & 0.139 & 0.141 \\
(711)-Fe3 & 0.144 & 0.146 & 0.148 & 0.149 \\
(711)-Si1 & 0.138 & 0.137 & 0.136 & 0.135 \\
(711)-Si3 & 0.141 & 0.139 & 0.137 & 0.135 \\
(711)-SiFe1 & 0.133 & 0.133 & 0.133 & 0.133 \\
(711)-SiFe & 0.150 & 0.150 & 0.150 & 0.150 \\
(711)-SiFe3 & 0.143 & 0.143 & 0.143 & 0.143 \\
(711)-Si5 & 0.144 & 0.140 & 0.137 & 0.133 \\
(711)-SiFe4 & 0.137 & 0.137 & 0.137 & 0.137 \\
\hline & & & &
\end{tabular}



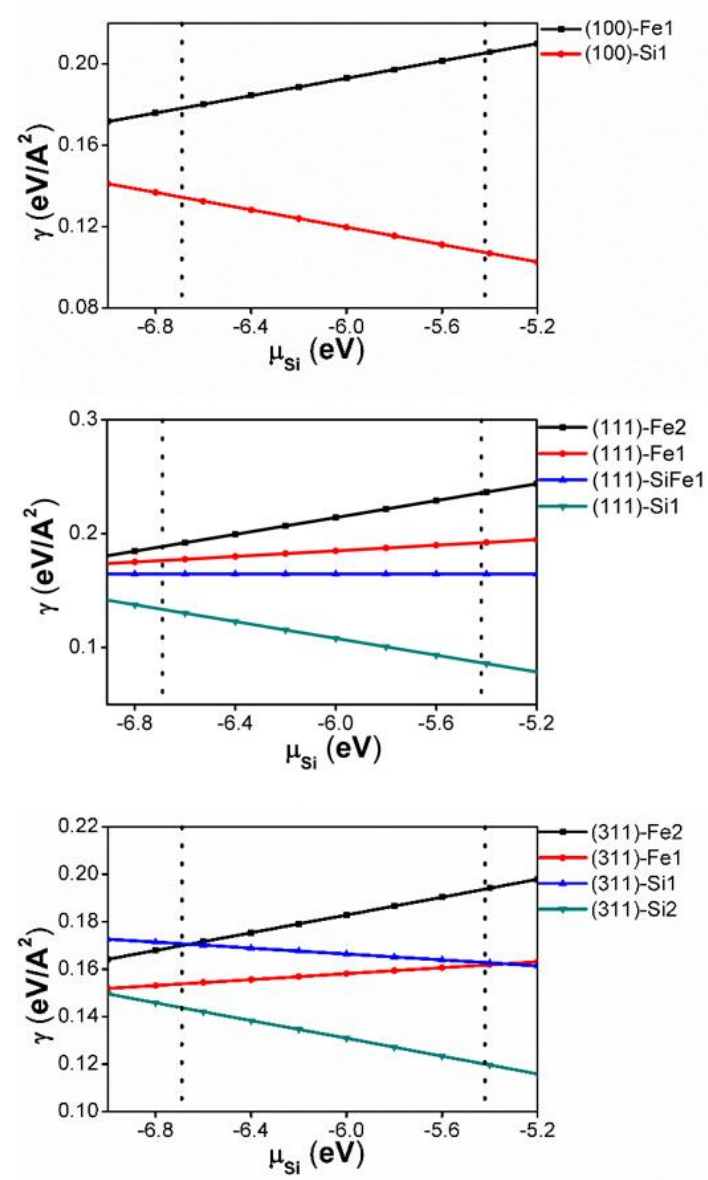
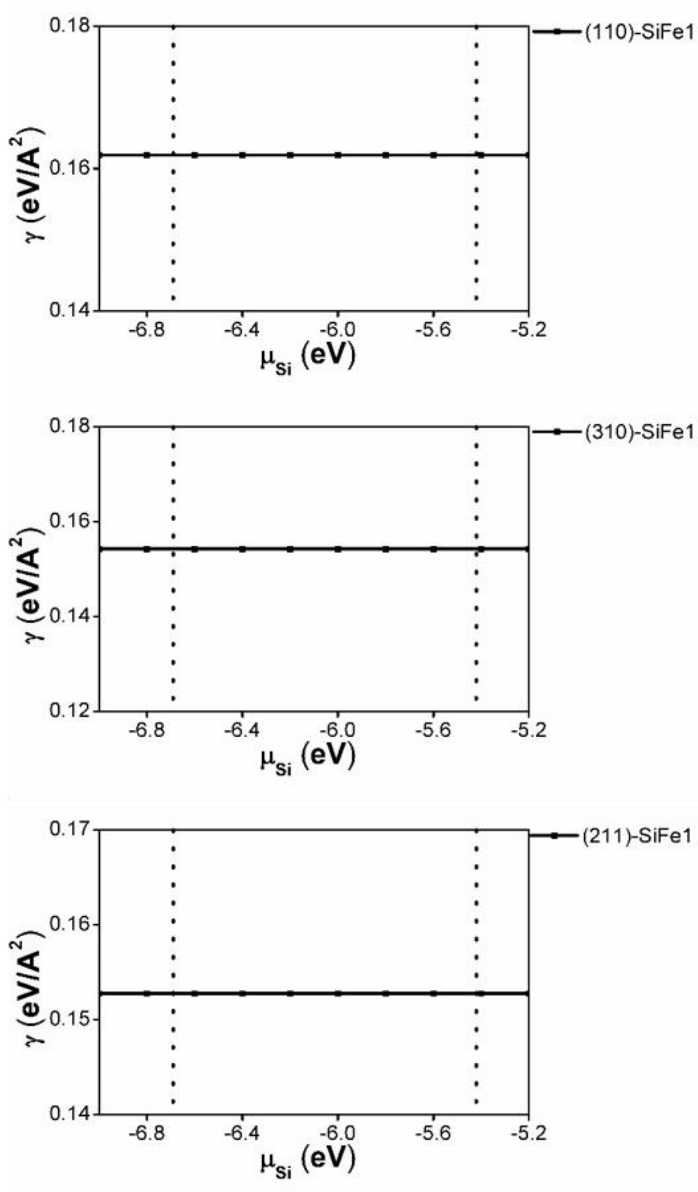

Figure S3. The relationship of surface energy of the $\mathrm{Fe}_{3} \mathrm{Si}$ surfaces with the different termination to the $\mu \mathrm{si}$. 

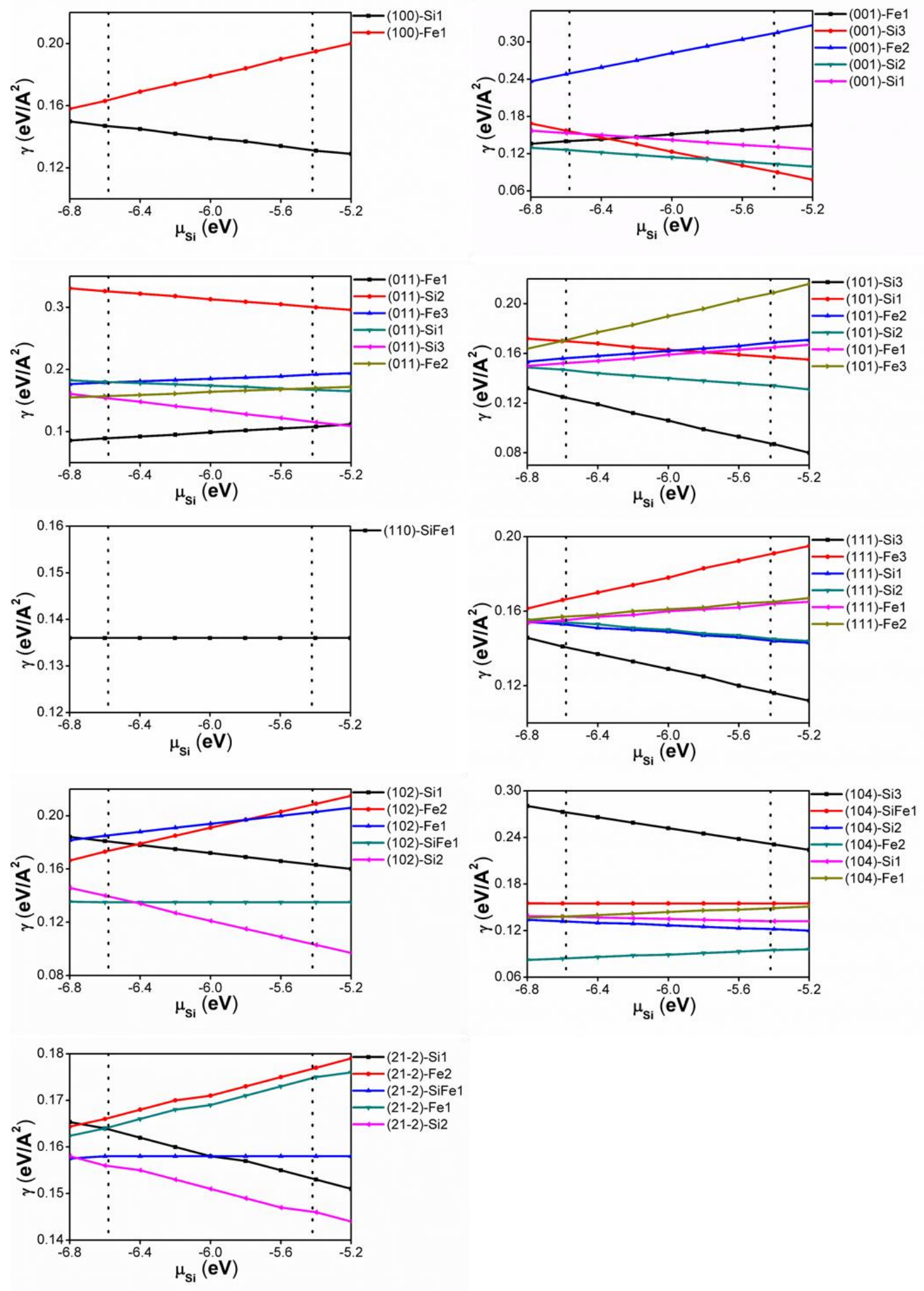

Figure S4. The relationship of surface energy of the $\mathrm{Fe}_{2} \mathrm{Si}$ surfaces with the different termination to the $\mu \mathrm{Si}$. 

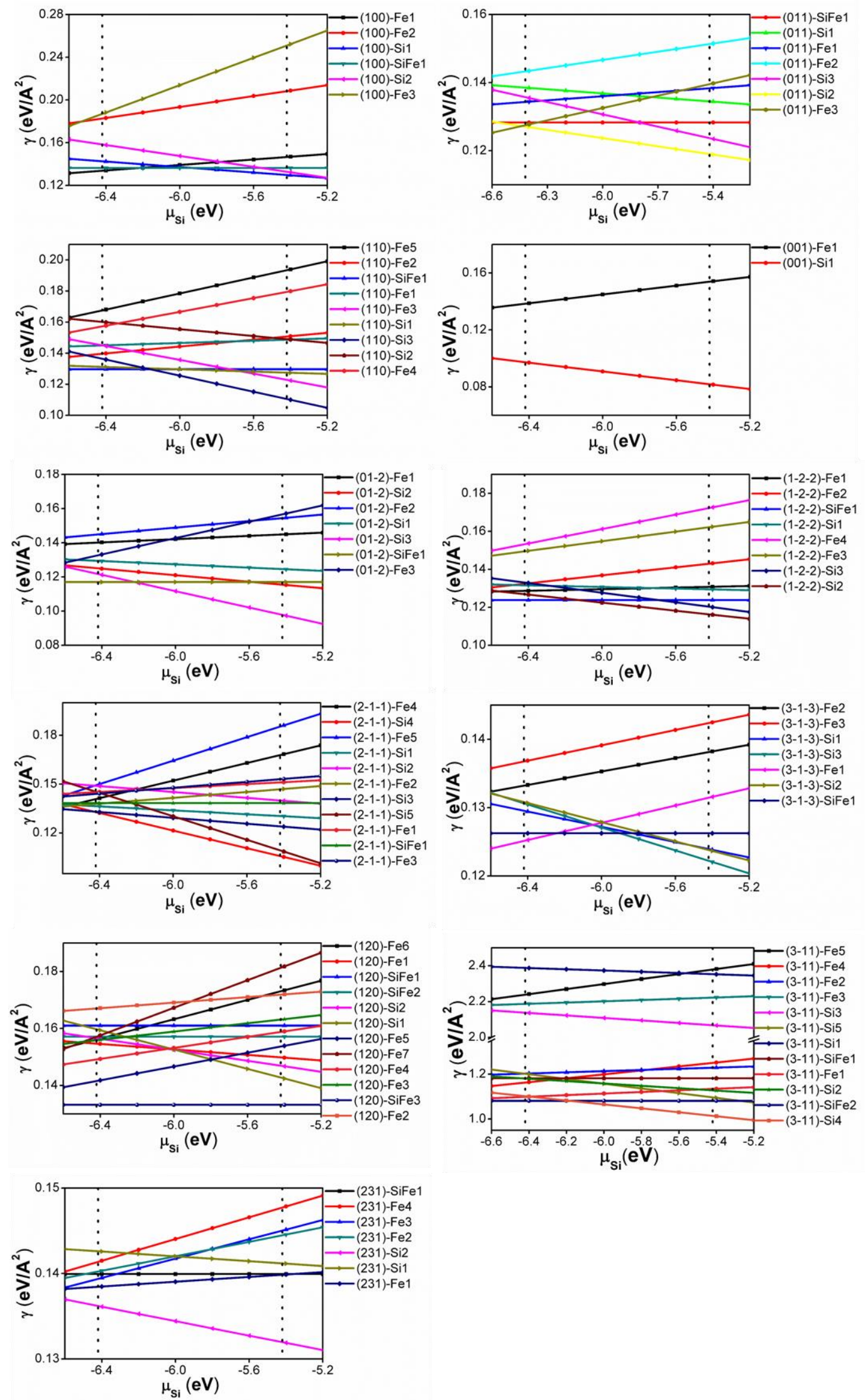

Figure S5. The relationship of surface energy of the $\mathrm{Fe}_{5} \mathrm{Si}_{3}$ surfaces with the different termination to the $\mu \mathrm{Si}$. 

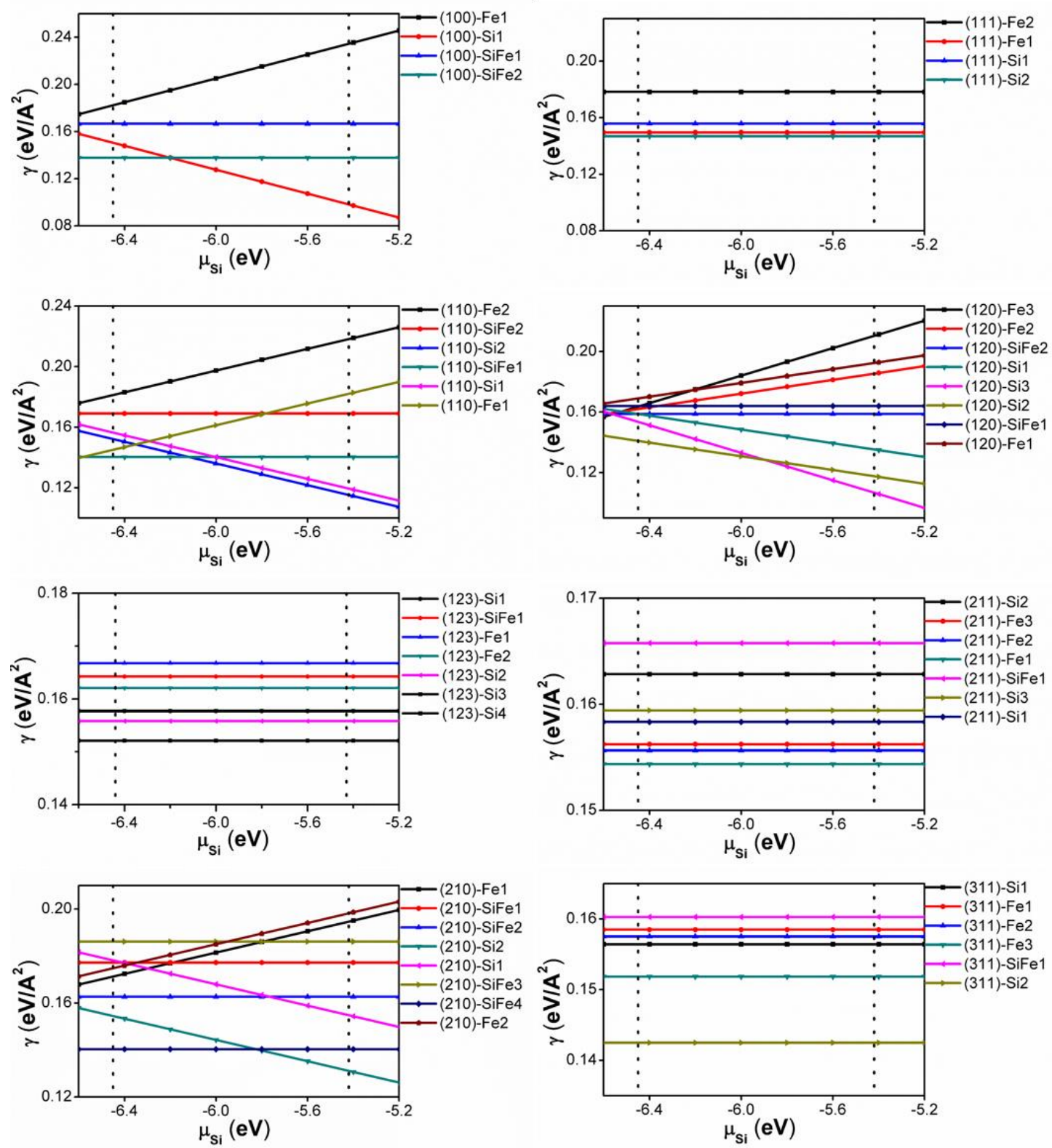

Figure S6. The relationship of surface energy of the FeSi surfaces with the different termination to the $\mu \mathrm{Si}$. 


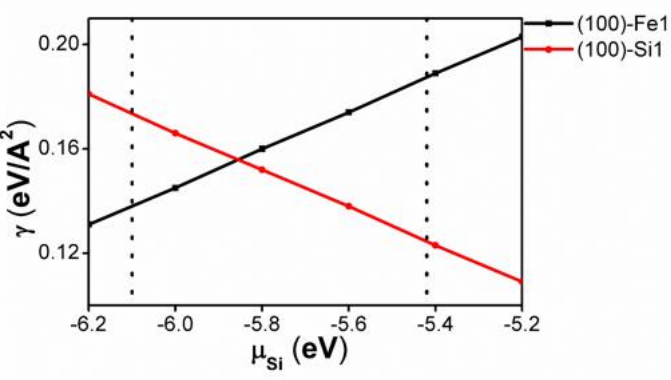

$0^{0.12}:{ }^{-(110)-S i F e}$
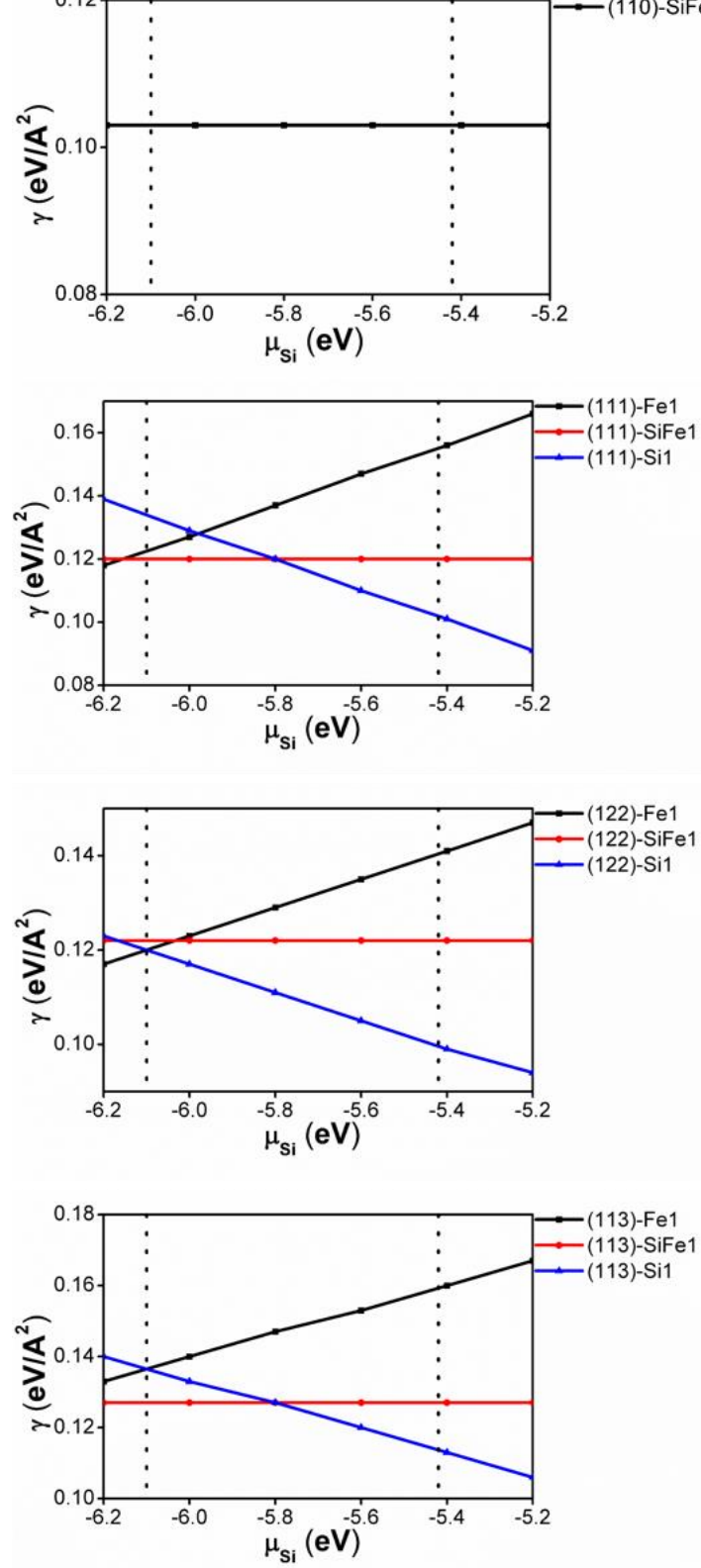
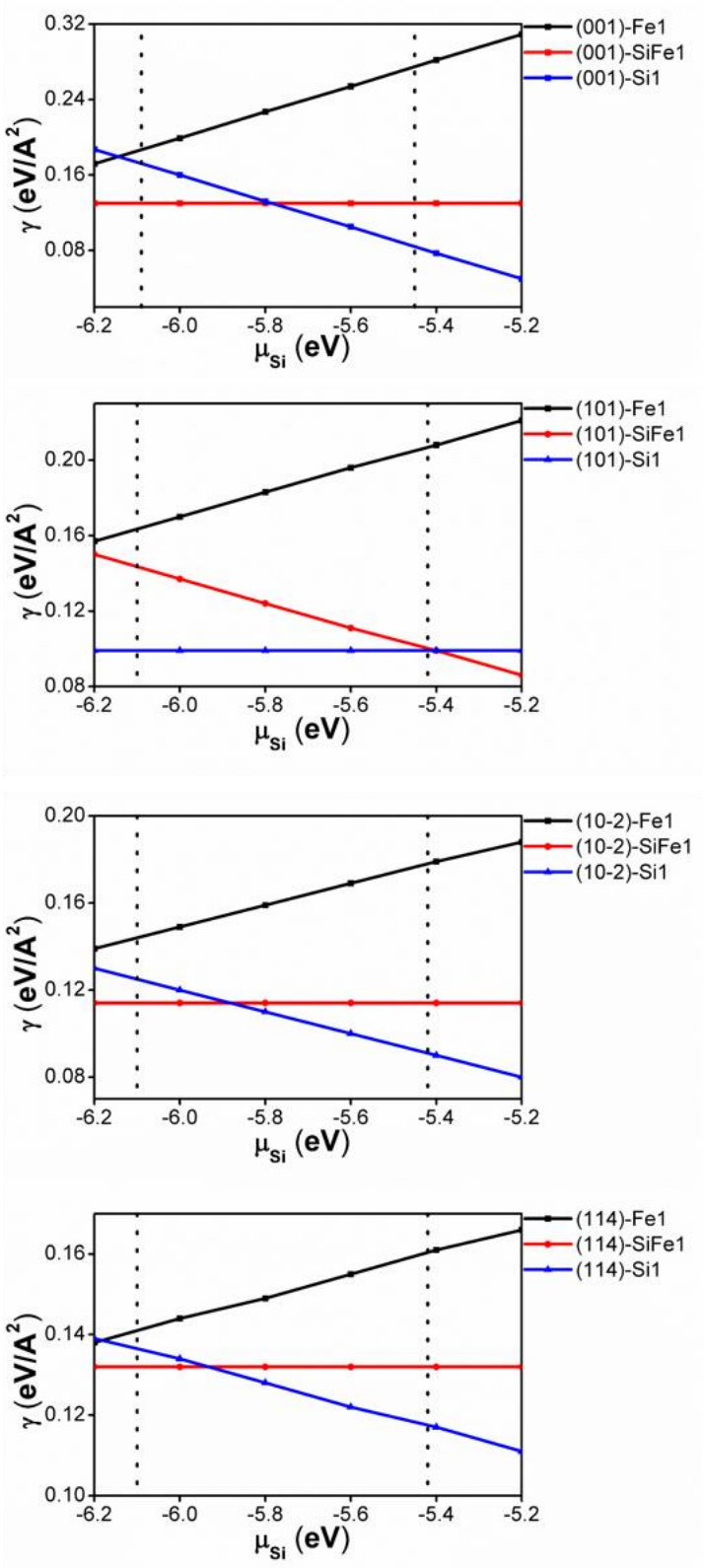

Figure S7. The relationship of surface energy of the $\alpha-\mathrm{FeSi}_{2}$ surfaces with the different termination to the $\mu \mathrm{Si}$. 

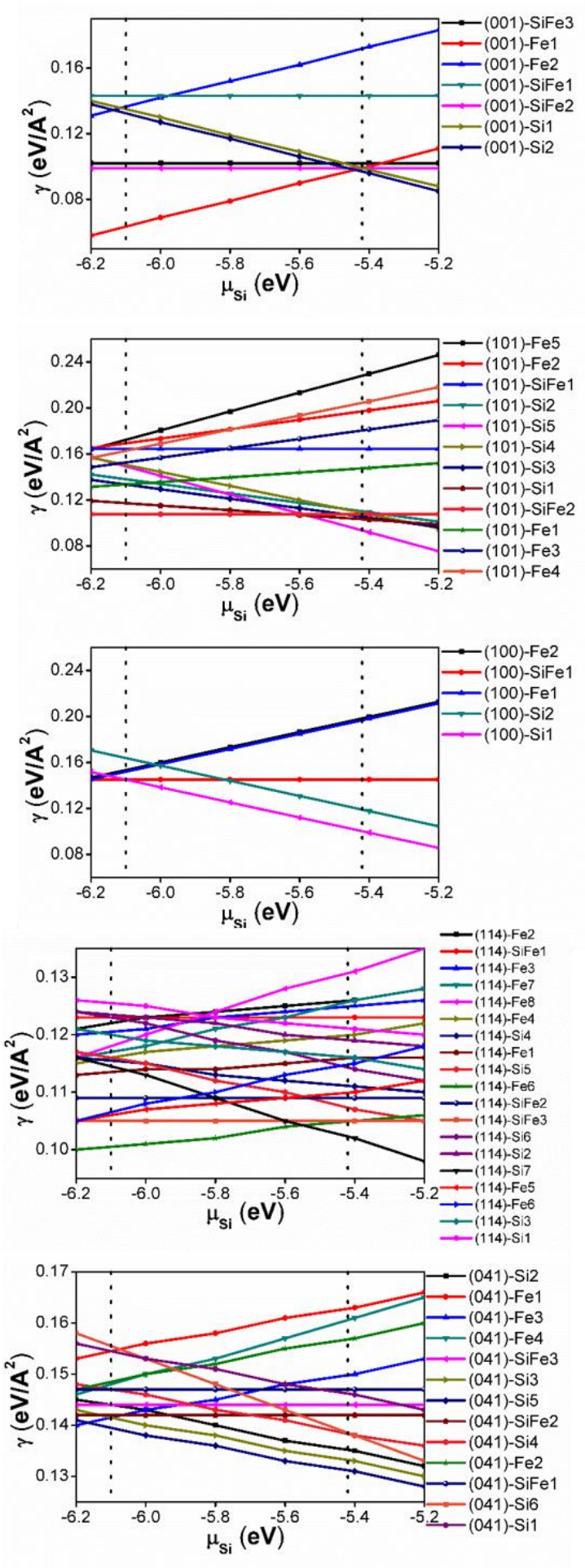
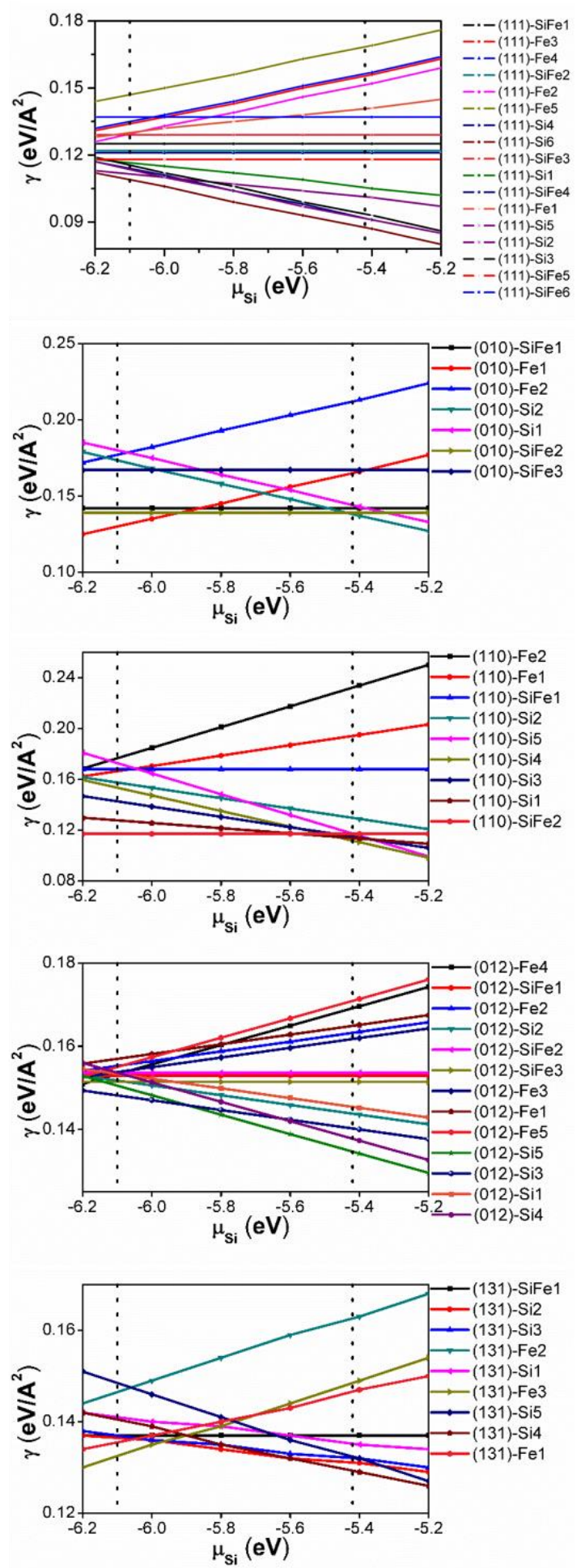

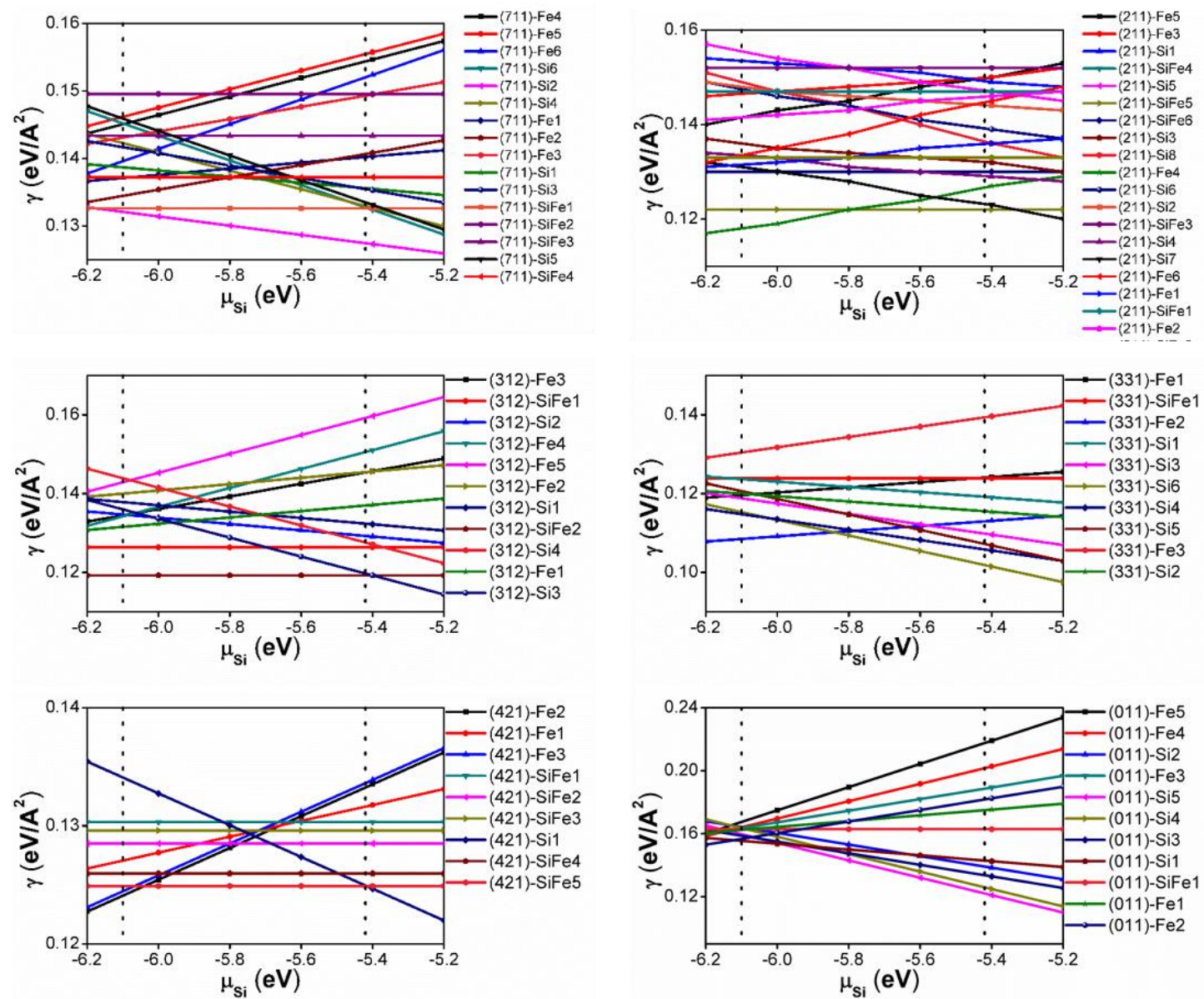

Figure S8. The relationship of surface energy of the $\beta-\mathrm{FeSi}_{2}$ surfaces with the different termination to the $\mu \mathrm{si}$.
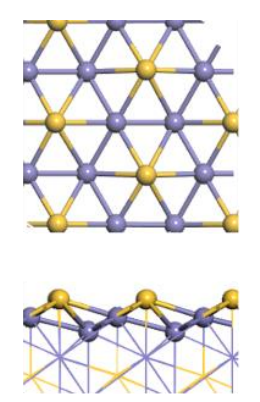

$\mathrm{Fe}_{3} \mathrm{Si}-(111)-\mathrm{Si1}$
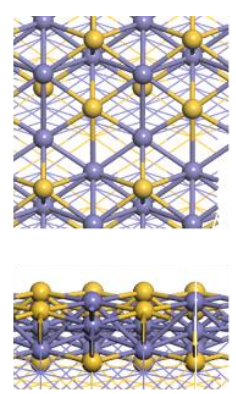

$\mathrm{Fe}_{2} \mathrm{Si}-(101)-\mathrm{Si} 3$
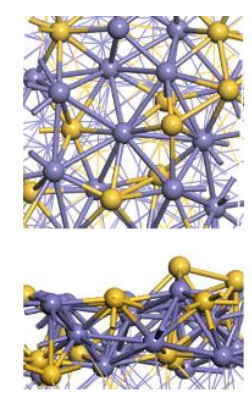

$\mathrm{Fe}_{5} \mathrm{Si}_{3}-(3-11)-\mathrm{Si} 4$
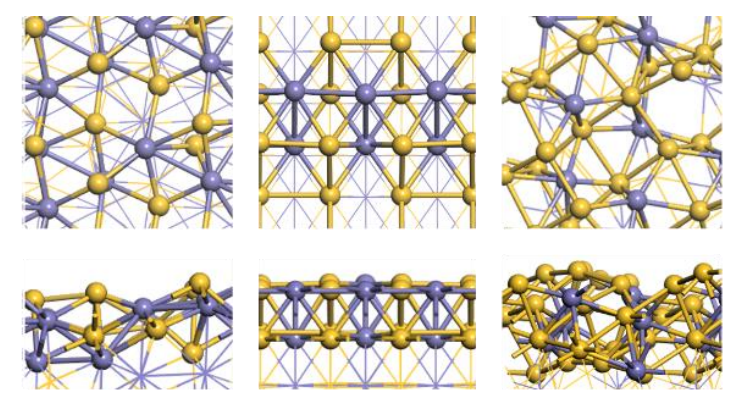

FeSi-(120)-Si3

$\alpha-\mathrm{FeSi}_{2}-(\mathbf{1 0 - 2 )}-\mathrm{Si} 1$

$\beta-\mathrm{FeSi}_{2}-(111)-\mathrm{Si} 6$

Figure S9. Surface structures of most stable surfaces which have largest exposed surface area in Wulff construction of the iron silicides 

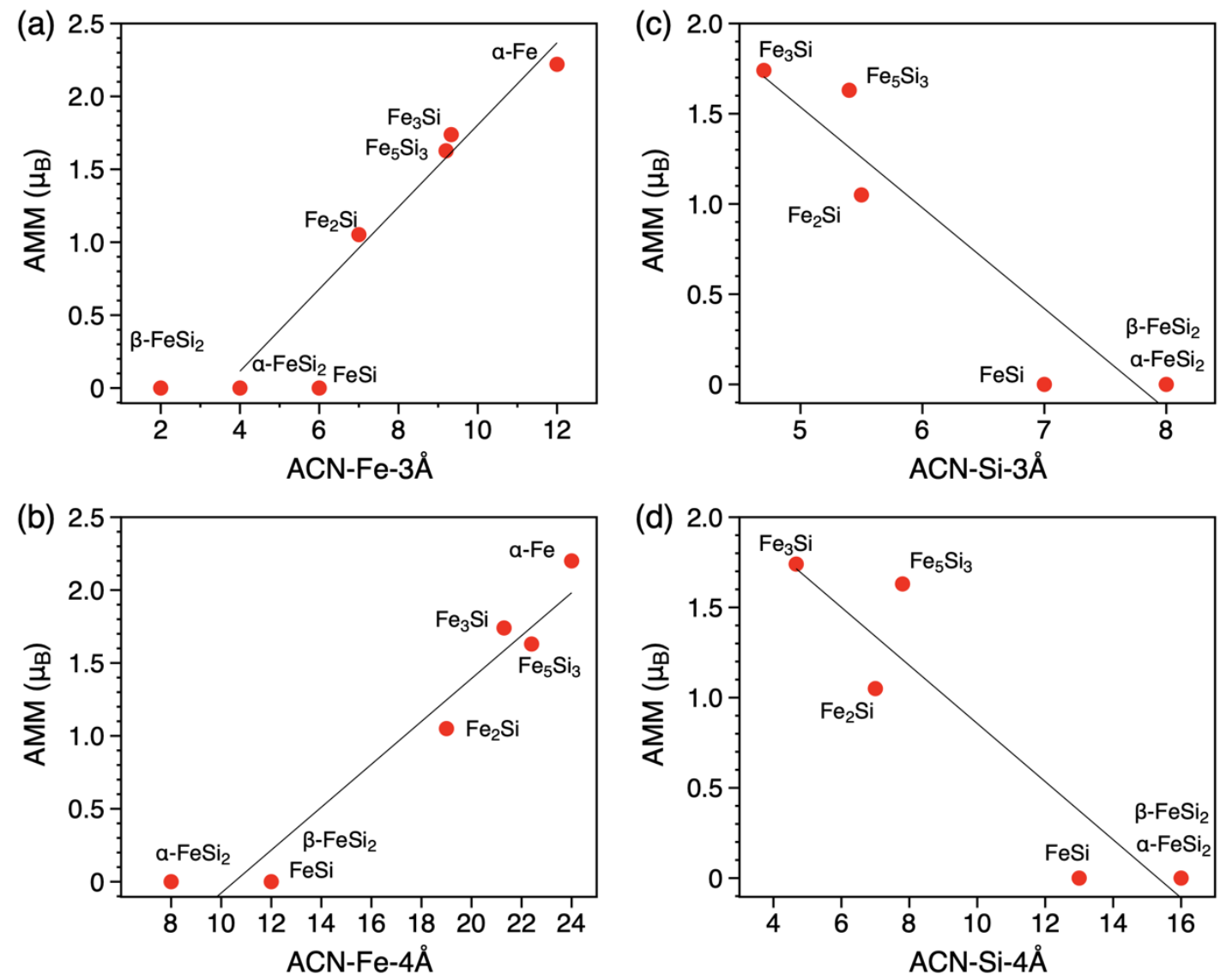

Figure S10 (a), (b) trend of average magnetic moment (AMM) of Fe with average number of coordinating Fe atoms (ACN-Fe) with different cutoff distance; (c), (d) trend of average magnetic moment (AMM) of Fe with average number of coordinating $\mathrm{Si}$ atoms (ACN-Si) with different cutoff distance.

The cutoff distance of $3 \AA$ is relatively small and only includes the first coordination sphere. Indeed, we also tested the effect of the second coordination sphere of Fe on the AMM-ACN correlation by using a larger cutoff distance of $4 \AA$. It turns out that for both AMM-(ACN-Fe) and AMM-(ACN-Si), the inclusion of the nextnearest neighbors slightly changes the positions of the data points, but doesn't alter too much of the general picture for the correlation (Figure S10). Therefore, the influence of the second coordination sphere of Fe atoms on the AMM-ACN correlation is secondary and can be neglected. In the main text, we consistently use a cutoff distance of $3 \AA$ to define the coordination spheres.

\section{Reference}

1. Kresse, G.; Furthmüller, J., Efficiency of Ab-Initio Total Energy Calculations for Metals and Semiconductors Using a Plane-Wave Basis Set. Comput. Mater. Sci. 1996, $6,15-50$.

2. Kresse, G.; Furthmüller, J., Efficient Iterative Schemes for Ab Initio Total-Energy 
Calculations Using a Plane-Wave Basis Set. Phys. Rev. B 1996, 54, 11169-11186.

3. Gunnarsson, O.; Lundqvist, B. I., Exchange and Correlation in Atoms, Molecules, and Solids by the Spin-Density-Functional Formalism. Phys. Rev. B 1976, 13, 42744298.

4. Perdew, J. P.; Yue, W., Accurate and Simple Density Functional for the Electronic Exchange Energy: Generalized Gradient Approximation. Phys. Rev. B 1986, 33, 88008802.

5. Perdew, J. P.; Wang, Y., Accurate and Simple Analytic Representation of the Electron-Gas Correlation Energy. Phys. Rev. B 1992, 45, 13244-13249.

6. Liang, Y. F.; Shang, S. L.; Wang, J.; Wang, Y.; Ye, F.; Lin, J. P.; Chen, G. L.; Liu, Z. K., First-Principles Calculations of Phonon and Thermodynamic Properties of Fe-Si Compounds. Intermetallics 2011, 19, 1374-1384.

7. Moroni, E. G.; Wolf, W.; Hafner, J.; Podloucky, R., Cohesive, Structural, and Electronic Properties of Fe-Si Compounds. Phys. Rev. B 1999, 59, 12860-12871.

8. Reuter, K.; Scheffler, M., Composition, Structure, and Stability of $\mathrm{RuO}_{2}$ (110) as a Function of Oxygen Pressure. Phys. Rev. B 2001, 65, 035406.

9. Reuter, K.; Scheffler, M., Composition and Structure of the $\mathrm{RuO}_{2}$ (110) Surface in an $\mathrm{O}_{2}$ and $\mathrm{CO}$ Environment: Implications for the Catalytic Formation of $\mathrm{CO}_{2}$. Phys. Rev. B 2003, 68, 045407.

10. White, R. T.; Espino-Rios, R. L.; Rogers, D. S.; Ring, M. A.; O'Neal, H. E., Mechanism of the Silane Decomposition. I. Silane Loss Kinetics and Rate Inhibition by Hydrogen. Ii. Modeling of the Silane Decomposition (All Stages of Reaction). Int. J. Chem. Kinet. 1985, 17, 1029-1065.

11. NIST-JANAF Thermochemical Tables Home Page. https://janaf.nist.gov/ (accessed Mar 18, 2019). 